\title{
Statistics of convective cloud turbulence from a comprehensive turbulence retrieval method for radar observations
}

Article

Accepted Version

Feist, M. M., Westbrook, C. D., Clark, P. A., Stein, T. H. M., Lean, H. W. and Stirling, A. J. (2019) Statistics of convective cloud turbulence from a comprehensive turbulence retrieval method for radar observations. Quarterly Journal of the Royal Meteorological Society, 145 (719). pp. 727-744. ISSN 1477870X doi: https://doi.org/10.1002/qj.3462 Available at https://centaur.reading.ac.uk/81215/

It is advisable to refer to the publisher's version if you intend to cite from the work. See Guidance on citing.

To link to this article DOI: http://dx.doi.org/10.1002/qj.3462

Publisher: Royal Meteorological Society

All outputs in CentAUR are protected by Intellectual Property Rights law, including copyright law. Copyright and IPR is retained by the creators or other copyright holders. Terms and conditions for use of this material are defined in the End User Agreement. 


\section{CentAUR}

Central Archive at the University of Reading

Reading's research outputs online 


\title{
Statistics of Convective Cloud Turbulence from a Comprehensive Turbulence
}

\author{
Retrieval Method for Radar Observations
}

\author{
Matthew M. Feist ${ }^{\mathrm{a}^{*}}$, Chris D. Westbrook ${ }^{\mathrm{a}}$, Peter A. Clark ${ }^{\mathrm{a}}$, Thorwald HM. Stein ${ }^{\mathrm{a}}$, \\ Humphrey W. Lean ${ }^{\mathrm{b}}$, Alison J. Stirling ${ }^{\mathrm{c}}$ \\ ${ }^{a}$ Department of Meteorology, University of Reading, Reading, UK. \\ ${ }^{b}$ MetOffice@Reading, University of Reading, Reading, UK. \\ ${ }^{c}$ Met Office, Exeter, UK.
}

${ }^{*}$ Correspondence to: M. M. Feist, Department of Meteorology, University of Reading, Reading, RG6 6BB, UK. E-mail: M.M.Feist@pgr.reading.ac.uk

1 Turbulent mixing processes are important in determining the evolution of convective clouds,

2 and the production of convective precipitation. However, the exact nature of these impacts

3 remains uncertain due to limited observations. Model simulations show that assumptions made

4 in parametrising turbulence can have a marked effect on the characteristics of simulated

5 clouds. This leads to significant uncertainty in forecasts from convection-permitting numerical weather prediction (NWP) models. This contribution presents a comprehensive method to retrieve turbulence using Doppler weather radar to investigate turbulence in observed clouds. This method involves isolating the turbulent component of the Doppler velocity spectrum width, expressing turbulence intensity as an eddy dissipation rate, $\varepsilon$. By applying this method throughout large datasets of observations collected over the southern UK using the $\left(0.28^{\circ}\right.$ beam-width) Chilbolton Advanced Meteorological Radar (CAMRa), statistics of convective 
cloud turbulence are presented. Two contrasting case days are examined: a shallow "shower" case, and a "deep convection" case, exhibiting stronger and deeper updrafts. In our observations, $\varepsilon$ generally ranges from $10^{-3}-10^{-1} \mathrm{~m}^{2} \mathrm{~s}^{-3}$, with the largest values found within, around and above convective updrafts. Vertical profiles of $\varepsilon$ suggest that turbulence is much stronger in deep convection; $95^{\text {th }}$ percentile values increase with height from $0.03-0.1$ $\mathrm{m}^{2} \mathrm{~s}^{-3}$, compared to approximately constant values of $0.02-0.03 \mathrm{~m}^{2} \mathrm{~s}^{-3}$ throughout the depth of shower cloud. In updraft regions on both days, the $95^{\text {th }}$ percentile of $\varepsilon$ has significant $(p<$ $10^{-3}$ ) positive correlations with the updraft velocity, and the horizontal shear in the updraft velocity, with weaker positive correlations with updraft dimensions. The $\varepsilon$-retrieval method presented considers a very broad range of conditions, providing a reliable framework for turbulence retrieval using high-resolution Doppler weather radar. In applying this method across many observations, the derived turbulence statistics will form the basis for evaluating the parametrisation of turbulence in NWP models.

Keywords: Radar; Doppler spectrum width; turbulence; convection; eddy dissipation rate; clouds.

\section{Introduction}

The effects of turbulence on the structure and evolution of convective clouds remain unclear in observations and numerical weather prediction (NWP) models. The turbulent entrainment of dry environmental air into cumulus clouds has long been known to play an important role in their growth and decay (Blyth, 1993). The specific location of entrained air can have a varied and substantial impact on resulting air motions within the cloud (Blyth et al., 1988). Turbulent mixing within clouds significantly impacts the microphysical processes governing the initiation of convective precipitation; the presence of turbulence accelerates cloud drop growth through increased rates of collision and coalescence (Grover and Pruppacher, 1985; Khain and Pinsky, 1995; Vohl et al., 1999; 


\section{Observing turbulence in convective clouds}

Falkovic et al., 2002; Pinksy and Khain, 2002). Although there is much evidence for the effects of turbulence on cloud processes, there remains uncertainty in their precise nature, and the implications for cloud evolution.

In recent years, regional numerical weather prediction (NWP) has improved to sufficient resolution that it is worthwhile abandoning the parametrisation of deep convective clouds, and, instead, allowing the unstable growth of explicit convective clouds. However, it is not feasible to forecast using resolutions sufficient to properly resolve all of the important features of the flow. Hence such models are known as 'convection-permitting models' (CPMs, Clark et al., 2016). Physical processes occurring on scales below those resolved in CPMs, such as turbulence, remain parametrised. CPMs generally adopt mixing-length-based turbulence closure schemes from LargeEddy Simulation (LES) models, such as the Smagorinsky-Lilly sub-grid scheme. It is not often clear whether the assumptions implicit in these schemes (such as the ability of the model to resolve an inertial sub-range of turbulence) are valid for CPMs, especially when using grid-lengths larger than $100 \mathrm{~m}$. Model simulations show that the configuration of turbulence parametrisations can have a profound effect on the characteristics of simulated clouds (e.g. Hanley et al., 2015). Until we advance our understanding of the effects of turbulence in observed clouds, justifiable attempts to evaluate and improve these parametrisations are difficult to make.

To improve our understanding of turbulence in observed clouds for model evaluation, observations of convective storm turbulence can be made with Doppler weather radar. By isolating the turbulent component to the Doppler velocity spectrum variance, near-instantaneous observations of turbulence can be made across large swathes of atmosphere. Turbulence retrieval with weather radar has clear benefits over using methods such as aircraft or ascent measurements which can only collect time-series information from single points in space. Radar retrieved fields of turbulence, expressed for convenience in terms of the dissipation rate of turbulent kinetic energy, $\varepsilon$, can be used to investigate relationships with storm strength and structure in a statistical sense for model evaluation. 


\section{Observing turbulence in convective clouds}

The accuracy to which $\varepsilon$ can be derived using the Doppler variance method is dependent on the accurate removal of variances associated with processes aside from inertial sub-range turbulence. Due to this somewhat indirect approach, evaluation of the Doppler variance method has previously been necessary through comparison of $\varepsilon$ estimates with in situ measurements and other radar retrieval techniques. Using Doppler weather radar, Labitt (1981) and Meischner et al. (2001) demonstrated good agreement between $\varepsilon$ derived from the Doppler variance method when compared with coordinated aircraft measurements in convective storms. Brewster and Zrnic (1986) found a high level of agreement between $\varepsilon$ from Doppler variance and $\varepsilon$ estimated from the "spatial spectra" method a method which involves taking the Fourier transform of a dataset of Doppler velocity measurements sampled either along a single ray at a given time, or at a fixed range gate over a period of time. Bouniol et al. (2003) performed a similar evaluation of the Doppler variance method using the spatial spectra method with a vertically-pointing Doppler cloud radar. Point-for-point comparison of $\varepsilon$ from the two methods showed a high level of agreement, especially for larger values. They concluded that the Doppler variance provides a reliable estimate of $\varepsilon$. The spatial spectra method itself has been evaluated by Shupe et al. (2012), who analysed Doppler velocity time series sampled with a vertically-pointing cloud radar in stratocumulus clouds. They found $\varepsilon$ estimates from spatial spectra to correspond well with aircraft and sonic anemometer measurements. Albrecht et al. (2016) examined cloud-top entrainment processes in non-precipitating stratocumulus using verticallypointing Doppler cloud radar. In this study, estimates of $\varepsilon$ were derived using both the Doppler spectrum variance and the Doppler velocity power spectrum (Fang et al., 2014), with good agreement found between the two methods. Methods to retrieve $\varepsilon$ at vertical incidence in (precipitating and nonprecipitating) stratocumulus using Doppler cloud radar are not well suited to retrieve $\varepsilon$ with scanning Doppler weather radar in precipitating convective clouds; as pertains to this study.

$$
\text { For scanning Doppler weather radar, the most significant contributor to Doppler variance aside }
$$
from turbulence is generally shear of the radial wind across the three dimensions of the beam (see Section 5), which requires careful separation from turbulence before estimates of $\varepsilon$ can be made. 


\section{Observing turbulence in convective clouds}

Melnikov and Doviak (2009) present a detailed method to retrieve $\varepsilon$ from the Doppler spectrum in vertical cross-sections through stratiform precipitation collected using an S-band Doppler weather radar. In their study, the radial and elevation shear components are calculated by least-squares fitting contiguous Doppler velocity measurements separately in each direction. A similar, though more sophisticated technique is applied in Section 5.3 to use linear regression to fit a 2-D linear velocity surface model (Neter and Wasserman, 1974) to Doppler velocities to evaluate shear over a spatial scale that we can specify and fix, guided by estimates of the inertial sub-range outer-scale (see Section 5.2). Using this method, we have been able to test the sensitivity of retrieved $\varepsilon$ to the scale over which shear is calculated and removed (Section 5.5).

Melnikov and Doviak (2009) calculated the azimuthal (transverse) shear from velocity gradients between two adjacent scans separated by $2^{\circ}$. They found variances from azimuthal shear to be small compared to elevation shears in stratiform clouds. However, stronger horizontal shears are likely to be found in the convective clouds analysed in this application; in particular, along the edges of updrafts (e.g. Istok and Doviak, 1986). In the present application, our radar data includes one scan performed through one azimuth per cloud. Consequently, we have developed new methods to estimate the azimuthal shear component from the radial shear alone (Section 5.4), allowing for its variance contribution to be estimated when adjacent scans are not available.

Generally, past studies focus on single storm cases when using radar methods to investigate convective storm turbulence (e.g. Brewster and Zrnic, 1986; Istok and Doviak, 1986). Often, the contributions to the Doppler spectrum width from mechanisms aside from turbulence are either purely assumed to be negligible, or are shown to be negligible only for the purpose of the application. As a result, a comprehensive method to retrieve $\varepsilon$ from radar fields under a wide range of conditions, and the statistical assessment of $\varepsilon$ that such a method permits, have not been presented. In developing this comprehensive approach, comparison is made with the more limited approaches that have appeared in the literature. 


\section{Observing turbulence in convective clouds}

In Sections $2-5$ of this paper, we present methods to accurately determine $\varepsilon$ from radar fields. This includes a summary of the conditions under which certain terms in the Doppler spectrum width equation can be neglected, and detailed methods for their calculation when they cannot. By applying this method across a dataset of radar observations, we have performed a statistical assessment of $\varepsilon$ in convective storms; this is presented in Section 6.

\section{Data and Methods}

\subsection{DYMECS - Radar observations with CAMRa}

This investigation follows on from the Dynamical and Microphysical Evolution of Convective Storms (DYMECS) project (Stein et al., 2014). The primary objective of DYMECS is to apply a statistical approach to investigate the dynamics, morphology and evolution of convective storms over southern England, both in radar observations and in high-resolution Met Office Unified Model (MetUM) simulations. An innovative track-and-scan method was used to obtain radar observations of hundreds of convective storms in 2011-2012. These were collected using the Chilbolton Advanced Meteorological Radar (CAMRa) located at the Chilbolton Observatory in Hampshire, UK. CAMRa is a $3 \mathrm{GHz}$ (S-band) Doppler weather radar with dual-polarisation capability. The 25-m diameter antenna provides an angular beam-width of $0.28^{\circ}$. The narrow beam provides elevation, $\theta$ and azimuthal, $\varphi$ resolutions of $100 \mathrm{~m}$ at $20 \mathrm{~km}$ range, and $500 \mathrm{~m}$ at $100 \mathrm{~km}$ range. In the radial direction, the pulse has a length of $75 \mathrm{~m}$, however, this is averaged to $300 \mathrm{~m}$ in our observations.

Observations were collected by scanning with CAMRa in two modes: elevation scanning with RHIs (range-height indicator) and azimuthal scanning with PPIs (plan-position indicator). By alternating between these two modes, detailed observations of hundreds of convective storms were collected on 40 days between July 2011 and August 2012. These observations have been compared 


\section{Observing turbulence in convective clouds}

with MetUM simulations to characterise storm morphology (Stein et al., 2014) and convective updraft characteristics (Nicol et al., 2015) in model and observations.

In Section 6, turbulence retrievals are analysed with corresponding fields of vertical velocity retrieved by Nicol et al. (2015) for DYMECS observations made on 20 April 2012 and 25 August 2012. These updraft velocities were estimated from the Doppler velocity by vertically integrating local changes in horizontal convergence under the assumption of flow continuity, accounting for the changes in density with height. The use of horizontal convergence to estimate vertical velocity removes the need to consider corrections for hydrometeor fall-speeds. The method required a zerovelocity boundary condition, either at the surface or cloud echo top. A weighted combination of velocity derived under both conditions was developed to minimise the vertical propagation of errors. In using only single-Doppler measurements, the omission of convergence in the direction perpendicular to the scanning plane led to a consistent under-estimation of the vertical velocity. To correct for this under-estimation, the suitable scaling for the vertical velocity was estimated from 500-m grid-length simulations of the MetUM for each case. These were made under assumptions that the simulated three-dimensional wind flows were suitably realistic and that the range of observed vertical velocities was represented in the model. The uncertainty in retrieved updraft velocities was estimated through point-for-point comparison of the scaled retrievals with model updrafts. For updraft velocities larger than $1 \mathrm{~m} \mathrm{~s}^{-1}$ (as analysed in this study), a root-mean-square difference of $2.5 \mathrm{~m} \mathrm{~s}^{-1}$ was found. It is likely that this uncertainty introduces scatter into the relationships between $\varepsilon$ and characteristics of updraft velocity presented in Section 6 , resulting in weaker measured correlations than may exist between $\varepsilon$ and the true updraft strength.

\subsection{Dissipation rates from CAMRa}

Doppler weather radar, such as CAMRa, can be used to infer characteristics of atmospheric turbulence from observations of the radial velocity field. The mean Doppler velocity $\bar{v}$, is the 


\section{Observing turbulence in convective clouds}

162

163

reflectivity-weighted average of radial point velocities found within a resolution volume (the volume of atmosphere observed by a single radar pulse, $V_{6}$ ). The Doppler spectrum variance $\sigma_{v}^{2}$, estimated by CAMRa, is the variance in the velocity of reflectors within $V_{6}$. Therefore, $\sigma_{v}^{2}$ includes velocity variance due to the turbulent motion of hydrometeors, among contributions from several other mechanisms. We assume that $\sigma_{v}^{2}$ can be decomposed into a sum of statistically independent variance contributions (Doviak and Zrnic, 1984).

$$
\sigma_{v}^{2}=\sigma_{s}^{2}+\sigma_{\mathrm{t}}^{2}+\sigma_{\mathrm{TV}}^{2}+\sigma_{\alpha}^{2}+\sigma_{\mathrm{o}}^{2}
$$

Where $\sigma_{v}^{2}$ has contributions primarily from radial wind shear across the sample volume $\sigma_{s}^{2}$, turbulence $\sigma_{\mathrm{t}}^{2}$, the distribution of hydrometeor fall-velocities $\sigma_{\mathrm{TV}}^{2}$, antenna rotation $\sigma_{\alpha}^{2}$, and hydrometeor oscillations $\sigma_{\mathrm{o}}^{2}$.

Using the theoretical framework presented by Frisch and Clifford (1974), we can calculate the eddy dissipation rate, $\varepsilon$ from $\sigma_{\mathrm{t}}^{2}$. Details of turbulent motion cannot be directly measured from $\sigma_{v}^{2}$. We can only infer $\sigma_{\mathrm{t}}^{2}$ from $\sigma_{v}^{2}$ by accounting for all other variance contributions in (1), either by subtracting their variance from $\sigma_{v}^{2}$, or by demonstrating that they are negligibly small compared to $\sigma_{\mathrm{t}}^{2}$

The eddy dissipation rate is the rate of energy transfer through the inertial sub-range of isotropic turbulence. For calculations of $\varepsilon$ to be accurate, $\sigma_{\mathrm{t}}^{2}$ must consist only of velocity variance due to eddies with a spatial scale less than the largest scale of the inertial sub-range, $\Lambda_{0}$. Ensuring this involves the careful separation of shear and turbulence, which is summarised in Section 5.

Once $\sigma_{\mathrm{t}}^{2}$ has been determined, $\varepsilon$ can be estimated from,

$$
\varepsilon \approx \frac{1}{\alpha}\left[\frac{\sigma_{\mathrm{t}}^{2}}{1.35 A\left(1-\frac{\gamma^{2}}{15}\right)}\right]^{\frac{3}{2}}
$$




\section{Observing turbulence in convective clouds}

$$
\varepsilon \approx \frac{1}{\beta}\left[\frac{\sigma_{\mathrm{t}}^{2}}{1.35 A\left(1+\frac{\xi^{2}}{15}\right)}\right]^{\frac{3}{2}}
$$

Where $\alpha$ (in metres) is the angular standard deviation of the two-way Gaussian beam pattern in the transverse (or elevation) direction (see $\sigma_{2}$ in Appendix S1), multiplied by the range from the radar. $\beta$ is the standard deviation of the pulse in the radial dimension (assumed uniform; for CAMRa $\beta=$ 26.25m). From this, $\gamma^{2}=1-\left(\frac{\beta}{\alpha}\right)^{2}$ and $\xi^{2}=1-\left(\frac{\alpha}{\beta}\right)^{2}$, and $A$ is the universal constant of inertial sub-range turbulence, with a value of 1.6.

If $\alpha>\beta$, then (2a) is used, with (2b) to be used if $\alpha<\beta$. This distinction has often been ignored in past studies, which typically employ a simplified version of (2a) to determine $\varepsilon$ (as stated in Doviak and Zrnic (1984)). For CAMRa, $\alpha>\beta$ at all ranges further than $17.9 \mathrm{~km}$ from the radar, so a similar approximation could be used. However, the application of (2a) and (2b) is straight-forward and any further approximation should be unnecessary.

Values of $\sigma_{v}^{2}$ generally range from $1-25 \mathrm{~m}^{2} \mathrm{~s}^{-2}$ in our observations. In reality, the negligibility of terms in (1) depends on their value relative to $\sigma_{\mathrm{t}}^{2}$, and as a result, no fixed variance value will always be negligibly small. Assuming that turbulence is only significant when $\sigma_{\mathrm{t}}^{2}>5 \mathrm{~m}^{2} \mathrm{~s}^{-2}$ (this translates to $\varepsilon>0.03 \mathrm{~m}^{2} \mathrm{~s}^{-3}$ when $\alpha=\beta$ ), we choose a negligibility threshold $\sigma_{\text {neg }}^{2}$, of $0.5 \mathrm{~m}^{2} \mathrm{~s}^{-2}$ for the purpose of this application. Whereby, variance contributions that are less than $\sigma_{\text {neg }}^{2}$ can be neglected. We can test the impact of this selection on $\varepsilon$ by determining the maximum combined variance of terms we may neglect. The variance contribution from $\sigma_{\alpha}^{2}$ is small enough to be ignored completely $\left(\sigma_{\alpha}^{2}<0.01 \mathrm{~m}^{2} \mathrm{~s}^{-2}\right.$, see Section 4). We can calculate $\sigma_{s}^{2}$ directly (Section 5), so no element of this contribution is neglected, regardless of value compared to $\sigma_{\text {neg. However, }}^{2}$ contributions from $\sigma_{\mathrm{TV}}^{2}$ and $\sigma_{\mathrm{o}}^{2}$ are not simple to measure directly in our observations. Contributions from $\sigma_{\mathrm{TV}}^{2}$ can be larger than $\sigma_{\mathrm{neg}}^{2}$ for rain and hail (Section 3), while $\sigma_{\mathrm{o}}^{2}$ is generally less than $0.25 \mathrm{~m}^{2} \mathrm{~s}^{-2}$ (Section 4). A maximum error would be incurred in $\sigma_{\mathrm{t}}^{2}$ of $0.75 \mathrm{~m}^{2} \mathrm{~s}^{-2}$ when neglecting 


\section{Observing turbulence in convective clouds}

$\sigma_{\mathrm{TV}}^{2}$ at $0.5 \mathrm{~m}^{2} \mathrm{~s}^{-2}$ (in the extreme case that hail or heavy rain is observed very close to the radar) and $\sigma_{\mathrm{o}}^{2}$ at $0.25 \mathrm{~m}^{2} \mathrm{~s}^{-2}$. If $\sigma_{\mathrm{t}}^{2}=5 \mathrm{~m}^{2} \mathrm{~s}^{-2}$, this would translate to a $21.6 \%$ positive error in $\varepsilon$. The error decreases as turbulence becomes more significant, to only $4.5 \%$ when $\sigma_{\mathrm{t}}^{2}=25 \mathrm{~m}^{2} \mathrm{~s}^{-2}$, and is independent of the range of the $\sigma_{\mathrm{t}}^{2}$ observation.

The range of $\varepsilon$ values we can estimate using the Doppler spectrum width technique is determined from the range of $\sigma_{v}^{2}$ values we can observe. This is related to the maximum ambiguous velocity interval (Nyquist velocity) of the radar. Keeler and Passarelli (1990) state that reliable measurements of the Doppler spectrum width can only be made between $0.02-0.2$ of the Nyquist interval. CAMRa has a Nyquist interval of $30 \mathrm{~m} \mathrm{~s}^{-1}$, so we can only reliably observe $\sigma_{v}$ between $0.6-6 \mathrm{~m} \mathrm{~s}^{-1}$, corresponding to $\sigma_{v}^{2}$ of $0.36-36 \mathrm{~m}^{2} \mathrm{~s}^{-2}$. In the case where $\sigma_{t}^{2}=\sigma_{v}^{2}$, we can determine the maximum detectable range in $\varepsilon$ from using this method with CAMRa. If observing such a range in $\sigma_{t}^{2}$ at a range of $50 \mathrm{~km}$, (the typical range of our storm observations), this would correspond to a maximum detectable range in $\varepsilon$ of $10^{-3}-1 \mathrm{~m}^{2} \mathrm{~s}^{-2}$.

The following three sections outline methods to assess the contribution of the non-turbulent terms in (1). By either calculating these terms directly, or showing that they are negligibly small compared to $\sigma_{\mathrm{t}}^{2}$, we can remove them from $\sigma_{v}^{2}$. This allows us to find $\sigma_{\mathrm{t}}^{2}$ as a residual velocity variance, and then convert this to $\varepsilon$ using $(2 a)$ and $(2 b)$.

\section{Doppler variance due to a distribution of hydrometeor fall velocities, $\sigma_{\mathrm{TV}}^{2}$}

\subsection{Theoretical framework and derivation of spectral variance equations}

In a given sample volume $V_{6}$, the presence of a distribution of hydrometeor diameters will lead to a distribution of hydrometeor fall velocities. In the circumstance where the radar beam is not perpendicular to hydrometeor velocity, this broadens the Doppler velocity spectrum. The observed variance contribution, $\sigma_{\mathrm{TV}}^{2}$ in (1), is at its maximum for a vertically pointing radar beam and decreases 


\section{Observing turbulence in convective clouds}

with angle from zenith. Values of $\sigma_{v}^{2}$ include the total variance of hydrometeor velocity within the pulse volume. According to (1), the variance in hydrometeor velocity from a fall-speed distribution $\left(\sigma_{\mathrm{TV}}^{2}\right)$ is statistically independent from the variance in hydrometeor velocity resulting from air motions within the cloud (included in $\sigma_{s}^{2}$ and $\sigma_{\mathrm{t}}^{2}$ ). Consequently, we require no assumptions regarding the vertical motion of air within the cloud when estimating $\sigma_{\mathrm{TV}}^{2}$.

Previous studies to estimate turbulence characteristics from Doppler velocity spectra typically assume $\sigma_{\mathrm{TV}}^{2}$ to be negligible (e.g. Frisch and Clifford, 1974; Chapman and Browning, 2001; Meischner et al., 2001; Melnikov and Doviak (2009)) unless observations were made at vertical incidence (Brewster and Zrnic, 1986). The expected variance due to $\sigma_{\mathrm{TV}}^{2}$ is reduced significantly by scanning at lower elevations (often the reason $\sigma_{\mathrm{TV}}^{2}$ is assumed negligible), however, this does not ensure the contribution is always negligibly small. Melnikov and Doviak (2009) neglected variance contributions from $\sigma_{\mathrm{TV}}^{2}$ purely by assuming they remained below $0.2 \mathrm{~m}^{2} \mathrm{~s}^{-2}$ when scanning at elevations below $20^{\circ}$ through stratiform precipitation. However, results presented in Section 3.2 suggest $\sigma_{\mathrm{TV}}^{2}$ from raindrops can reach $1 \mathrm{~m}^{2} \mathrm{~s}^{-2}$ when scanning at $20^{\circ}$, though this remains dependent on radar reflectivity. The objectives of this section are to: provide a means to estimate $\sigma_{\mathrm{TV}}^{2}$ when required, provide justification when neglecting $\sigma_{\mathrm{TV}}^{2}$ contributions (showing that $\sigma_{\mathrm{TV}}^{2}<\sigma_{\text {neg }}^{2}$ ), and inform how future scanning strategies for turbulence retrieval can be tailored to ensure $\sigma_{\mathrm{TV}}^{2}$ is always negligible.

For application to RHI radar observations, we classify two hydrometeor types based on the height of the $0^{\circ} \mathrm{C}$ isotherm, $z_{0^{\circ}} \mathrm{C}$, which is estimated from the location of bright-band radar reflectivity in our observations. Though $z_{0}{ }^{\circ} \mathrm{C}$ varies for different DYMECS case days, the average height is $\sim 1.5$ $\mathrm{km}$. For simplicity, we assume any reflectivity returned from below this level is due to liquid raindrops, and any reflectivity from above is due to ice aggregates. By making this simple distinction, we can estimate $\sigma_{\mathrm{TV}}^{2}$ in all areas of an RHI scanning domain. In addition to aggregates and raindrops, graupel and hail are also important hydrometeor types, especially in convective clouds. We therefore 


\section{Observing turbulence in convective clouds}

255

256

257

258

259

extend our analysis to assess the impact of hail, and consider the effects of graupel (treated as lowdensity hailstones) in Section 3.3.

We assume the reflectivity for a given $V_{6}$ is dominated by one hydrometeor type, and that hydrometeors are falling vertically downwards at terminal velocity relative to the vertical air motions within the cloud. We assume hailstones are dry and are of solid ice with homogeneous density. For hydrometeor mass calculations, we assume that both raindrops and hailstones are spherical.

To estimate the relative size of $\sigma_{\mathrm{TV}}^{2}$ when compared to $\sigma_{v}^{2}$, we can characterise $\sigma_{\mathrm{TV}}^{2}$ as the variance of the reflectivity-weighted mean fall velocity in $V_{6}$ as,

$$
\sigma_{\mathrm{TV}_{j}}^{2}={\overline{W_{J}^{2}}}_{-\bar{W}_{j}^{2}}
$$

where $\sigma_{T V_{j}}^{2}$ has units $\mathrm{m}^{2} \mathrm{~s}^{-2}, W$ is the reflectivity-weighted hydrometeor fall velocity, and $j$ refers to the hydrometeor type. We estimate $\overline{W_{J}^{2}}$ and $\bar{W}_{j}^{2}$ by evaluating the following integrals,

$$
\begin{gathered}
\overline{W_{j}^{2}}=\frac{\int_{0}^{\infty} V_{j}(D)^{2} M_{j}(D)^{2} n_{j}(D) d D}{\int_{0}^{\infty} M_{j}(D)^{2} n_{j}(D) d D}, \\
\bar{W}_{j}^{2}=\left(\frac{\int_{0}^{\infty} V_{j}(D) M_{j}(D)^{2} n_{j}(D) d D}{\int_{0}^{\infty} M_{j}(D)^{2} n_{j}(D) d D}\right)^{2},
\end{gathered}
$$

where $V_{j}(D), M_{j}(D)$ and $n_{j}(D)$ are terminal velocity-diameter, mass-diameter and particle-size distribution (DSD) relationships for hydrometeor $j$, respectively, and $D$ is the hydrometeor diameter in metres.

In (4) and (5), we assume that particle reflectivity is proportional to $M_{j}(D)^{2}$. We are in the Rayleigh scattering regime, and hence this is a reasonable assumption for a $3 \mathrm{GHz}$ radar. The integral $R_{j} \int_{0}^{\infty} M_{j}(D)^{2} n_{j}(D) d D$ provides the radar reflectivity in $\mathrm{mm}^{6} \mathrm{~m}^{-3}$. The term $R_{j}$ is cancelled out in (4) and (5), but is given by, 


\section{Observing turbulence in convective clouds}

$$
R_{j}=10^{18} \frac{\left|K_{j}\right|^{2}}{\left|K_{\text {water }}\right|^{2}}\left(\frac{6}{\pi \rho_{j}}\right)^{2}
$$

276 where $\left|K_{j}\right|^{2}$ and $\rho_{j}$ are the dielectric factor and density of hydrometeor $j$.

$$
V_{j}(D)=p_{j} D^{q_{j}}
$$

where $V$ is the fall velocity and $D$ is the drop diameter. For ice aggregates, $D$ is the melted diameter. Values of $p$ and $q$ for raindrops, ice aggregates and hailstones are taken from Atlas and Ulbricht (1977), Gunn and Marshall (1958) and Cheng and English (1982), respectively. These have been converted into S. I. units (See Table 1).

The hydrometeor mass $M$, as a function of particle diameter $D$, can be expressed in the form,

$$
M_{j}(D)=a_{j} D^{b_{j}}
$$

where $M$ and $D$ are in S. I. units.

We assume that the DSD of each hydrometeor class can be approximated by an exponential distribution of form given by Marshall and Palmer (1948).

$$
n_{j}(D)=N_{0_{j}} \exp \left(-\lambda_{j} D\right)
$$

Where $N_{0_{j}}$ and $\lambda_{j}$ are the intercept $\left(n_{j}(D=0)\right)$ and slope parameters, respectively, for hydrometeor type $j$. We consider this a suitable approximation; spectral broadening owing to a distribution in fall velocity has been shown to be nearly independent of the precise shape of the size distribution (Lhermitte, 1963).

For rain and ice aggregates, values of $\rho,|K|^{2}, a, b$ and $N_{0}$, are sourced from the UK Met Office 


\section{Observing turbulence in convective clouds}

294

295

296

297

298

299

300

301

302

303

304

305

306

307

308

309

310

311

312

313

314

Unified Model microphysics scheme, as summarised in Stein et al. (2014) (See Table 1). For hail, we use an $N_{0}$ of $1.2 \times 10^{4} \mathrm{~m}^{-4}$ taken from Waldvogel et al. (1978). The sensitivity of $\sigma_{\mathrm{TV}_{j}}^{2}$ to $N_{0_{j}}$ is discussed in Section 3.3.

To evaluate (3), we first substitute (7) - (9) into (4) and (5) using values from Table 1. By using a gamma function solution for the integrals in (3) we derive expressions for Doppler spectral variance contribution for the three hydrometeor varieties. At this point, they are functions only of DSD parameter, $\lambda_{j}$. Stein et al. (2014) provide an expression relating $\lambda_{j}$ to radar reflectivity, $Z_{j}$,

$$
\lambda_{j}=\left(\frac{R_{j} a_{j}^{2} N_{0_{j}} \Gamma\left(1+2 b_{j}\right)}{Z_{j}}\right)^{\frac{1}{1+2 b_{j}}}
$$

where $Z_{j}$ is the radar reflectivity of hydrometeor $j$ and has linear units of $\mathrm{mm}^{6} \mathrm{~m}^{-3}$.

Substituting (10) into the $\sigma_{\mathrm{TV}_{j}}^{2}\left(\lambda_{j}\right)$ expressions and simplifying using values from Table 1 , produces spectral variance equations for rain, ice aggregates and hail,

$$
\begin{aligned}
& \sigma_{\mathrm{TV}_{\text {rain }}}^{2}=0.62 Z^{0.191} \sin ^{2} \theta_{\mathrm{el}}, \\
& \sigma_{\mathrm{TV}_{\mathrm{agg}}}^{2}=0.029 Z^{0.119} \sin ^{2} \theta_{\mathrm{el}}, \\
& \sigma_{\mathrm{TV}_{\text {hail }}}^{2}=1.7 Z^{0.143} \sin ^{2} \theta_{\mathrm{el}},
\end{aligned}
$$

Where $Z$ is in $\mathrm{mm}^{6} \mathrm{~m}^{-3}, \sigma_{\mathrm{TV}_{j}}^{2}$ has units of $\mathrm{m}^{2} \mathrm{~s}^{-2}$ and $\theta_{\mathrm{el}}$ is the elevation angle of the reflectivity observation measured from the surface. Together, these expressions can be used to estimate the Doppler variance contribution due to the distribution of hydrometeor fall speeds in $V_{6}$.

\subsection{Analysis of $\sigma_{\mathrm{TV}_{j}}^{2}$}

Reflectivity measurements in our observations with CAMRa are generally no less than $-20 \mathrm{dBZ}$ (the minimum detectable echo at $10 \mathrm{~km}$ range), and no more than $60 \mathrm{dBZ}$. In our application, we 


\section{Observing turbulence in convective clouds}

therefore classify $\sigma_{\mathrm{TV}_{j}}^{2}\left(Z_{j}=-20 \mathrm{dBZ}\right)$ and $\sigma_{\mathrm{TV}_{j}}^{2}\left(Z_{j}=60 \mathrm{dBZ}\right)$ as the minimum and maximum potential variances we encounter from each hydrometeor type.

Equations (11) - (13) show that $\sigma_{\mathrm{TV}_{j}}^{2}$ increases with radar reflectivity and elevation angle of observation. Assuming a vertically pointing radar beam, and given $Z_{j}$ in the range of $-20 \mathrm{dBZ}$ to 60 $\mathrm{dBZ}, \sigma_{\mathrm{TV}}^{2}$ rain increases from 0.26 to $8.62 \mathrm{~m}^{2} \mathrm{~s}^{-2}, \sigma_{\mathrm{TV}_{\text {agg }}}^{2}$ from 0.02 to $0.15 \mathrm{~m}^{2} \mathrm{~s}^{-2}$ and $\sigma_{\mathrm{TV}_{\text {hail }}}^{2}$ from 0.90 to $12.53 \mathrm{~m}^{2} \mathrm{~s}^{-2}$. For DYMECS observations, RHIs scanned at a maximum elevation angle of $15^{\circ}$. Figure 1a displays (11) - (13) for a vertically pointing beam (black lines) and for $15^{\circ}$ elevation (grey lines). Compared with a vertically pointing beam, if $Z_{j}$ is sampled at $15^{\circ}$ elevation, values of $\sigma_{\mathrm{TV}_{j}}^{2}$ are respectively reduced by a factor of 14 .

A maximum $\sigma_{\mathrm{TV}}^{2}$ agg of $0.15 \mathrm{~m}^{2} \mathrm{~s}^{-2}$ suggests that the contribution from ice aggregates is always less than $\sigma_{\text {neg. }}^{2}$. Assuming that ice aggregates constitute all hydrometeors above $z_{0}{ }^{\circ} \mathrm{C}$, then $\sigma_{\mathrm{TV}}^{2}$ is negligible for all observations made above this level. For rain observations, which we assume are limited to below $z_{0^{\circ} \mathrm{C}}$, the equivalent maximum of $8.62 \mathrm{~m}^{2} \mathrm{~s}^{-2}$ is comparably large, and so $\sigma_{\mathrm{TV}}^{2}$ rain cannot always be neglected. Using (11), we see $\sigma_{\mathrm{TV}_{\text {rain }}}^{2}(60 \mathrm{dBZ})<\sigma_{\text {neg }}^{2}$ for all rain observed at $\theta_{\mathrm{el}}<$ $13.9^{\circ}$.

Under the circumstances that: $\sigma_{\mathrm{TV}_{\mathrm{agg}}}^{2}$ is always negligible, $\sigma_{\mathrm{TV}}^{2}$ rain is negligible when $\theta_{\mathrm{el}}<13.9^{\circ}$, $z_{0^{\circ} \mathrm{C}}$ can be estimated, and hail is not present, we can describe the negligibility of $\sigma_{\mathrm{TV}}^{2}$ in terms of a minimum range from the radar, $R_{\min }$. For our application, $R_{\min }$ is simply the range from the radar a pulse reaches a height of $z_{0^{\circ} \mathrm{C}}$ when transmitted at $\theta_{\mathrm{el}}<13.9^{\circ}$. In our RHI observations (where $\left.z_{0^{\circ} \mathrm{C}} \sim 1.5 \mathrm{~km}\right), \sigma_{\mathrm{TV}}^{2}$ is negligibly small everywhere at ranges further than $6.1 \mathrm{~km}$ from the radar. While $\sigma_{\mathrm{TV}}^{2}$ can still be significant due to rain occurring nearer than $R_{\min }$, below $z_{0^{\circ} \mathrm{C}}$, it remains conditional on both $Z_{\text {rain }}$ and $\theta_{\mathrm{el}}$. Observations used in this application were rarely made closer than $30 \mathrm{~km}$ from the radar, and so we neglect $\sigma_{\mathrm{TV}}^{2}$ for rain and ice aggregates. 


\section{Observing turbulence in convective clouds}

According to (13), hail can contribute more to $\sigma_{v}^{2}$ than rain. However, hail is generally a much less common, more localised occurrence than rain. As a result, the detection of hail using retrieved radar parameters (e.g. hail differential reflectivity $H_{\mathrm{DR}}$, Depue et al. (2007)) is necessary before (13) can be reliably applied. If observations do indeed include hail, (13) suggests that $\sigma_{\mathrm{TV}}^{2}(60 \mathrm{dBZ})$ falls below $\sigma_{\text {neg }}^{2}$ for all hail observations made at $\theta_{\mathrm{el}}<11.5^{\circ}$. Due to the potential for hail presence both above and below $z_{0^{\circ} \mathrm{C}}$, negligibility based on range from radar is not stated. However, as the minimum range of observations was $30 \mathrm{~km}$, hail would need to be observed at $6 \mathrm{~km}$ altitude for $\sigma_{\mathrm{TV}}^{2}$ hail to exceed $\sigma_{\text {neg, }}^{2}$, which is unlikely to have occurred.

Based on our threshold for negligibility $\sigma_{\text {neg }}^{2}$, the estimation of $z_{0^{\circ} \mathrm{C}}$, and under the assumptions made in the derivation of (11) - (13), we can neglect variance contributions from $\sigma_{\mathrm{TV}}^{2}$ in our observations. Due to the dependence of $(11)-(13)$ only on $Z$ and $\theta_{\text {el }}$, we expect this conclusion to hold true for other scanning weather radars.

\subsection{Sensitivity of $\sigma_{\mathrm{TV}_{\text {rain }}}^{2}$ and $\sigma_{\mathrm{TV}_{\text {hail }}}^{2}$ to assumptions}

In this section, we examine the sensitivity of our results to some of the assumptions made in the derivation of (11) and (13). For ice aggregates, no reasonable sensitivity testing has resulted in the factor 3 increase in $\sigma_{\mathrm{TV}}^{2}$ agg required to even conditionally exceed $\sigma_{\mathrm{neg}}^{2}$. As a result, sensitivity tests involving ice aggregates have been omitted from this discussion, and we conclude that $\sigma_{\mathrm{TV}}^{2}$ isg always negligible.

For rain and hail, we expect little uncertainty in the majority of values in Table 1 . The first potential source of uncertainty lies with the treatment of hail as dry with the density of solid ice. We compare $\sigma_{\mathrm{TV}}^{2}$ hail when hailstones are dry with the density of solid ice (assumed in (13)), to lowdensity and melting hailstones. Melting hailstones will possess a thin outer layer of liquid water, appearing to the radar as large raindrops. To simulate this effect, we change the dielectric factor 


\section{Observing turbulence in convective clouds}

362

363

$\left|K_{\text {hail }}\right|^{2}$, in (6) from 0.174 to 0.93 . Resulting variance contributions are $21 \%$ lower than for dry hailstones for any given reflectivity. Assuming all hailstones below $z_{0^{\circ} \mathrm{C}}$ have a liquid water layer, this reduction leads to $\sigma_{\mathrm{TV}_{\text {hail }}}^{2}(60 \mathrm{dBZ}) \approx \sigma_{\mathrm{TV} \text { rain }}^{2}(60 \mathrm{dBZ})$ below $z_{0^{\circ} \mathrm{C}}$. For observations made below $z_{0}{ }^{\circ} \mathrm{C}=1.5 \mathrm{~km}$, we find that $\sigma_{\mathrm{TV}}^{2}<\sigma_{\text {neg }}^{2}$ at all ranges further than $6.5 \mathrm{~km}$ from the radar, regardless of hydrometeor type. If we further consider melting hailstones consisting of low-density ice that is more consistent with graupel $\left(\rho_{\text {hail }}=500 \mathrm{~kg} \mathrm{~m}^{-3}\right)$, this leads to a combined reduction in $\sigma_{\mathrm{TV}}^{2}$ hail of $34 \%$, at which point $\sigma_{\mathrm{TV}}^{2}$ hail $(60 \mathrm{dBZ})<\sigma_{\mathrm{TV} \text { rain }}^{2}(60 \mathrm{dBZ})$, and we revert to neglecting $\sigma_{\mathrm{TV}}^{2}$ at ranges further than $6.1 \mathrm{~km}$.

A second source of uncertainty lies with the chosen values of $N_{0}$; respective values for rain and hail are assumed constant. For rain, we use $N_{0_{\text {rain }}}=8 \times 10^{6} \mathrm{~m}^{-4}$ from Marshall and Palmer (1948), who demonstrate its independence of rainfall intensity. The assumption of a constant $N_{0_{\text {hail }}}$ is not as safe as for raindrops as it depends on the largest hail diameter, $D_{\text {max }}$, and has been shown to vary from $10^{3}-10^{5} \mathrm{~m}^{-4}$ (Ulbricht, 1974). Our chosen value of $N_{0_{\text {hail }}}=1.2 \times 10^{4} \mathrm{~m}^{-4}$ from Waldvogel et al. (1978) is roughly in the centre of this range, and is very similar to values of $1.1-$ $1.4 \times 10^{4} \mathrm{~m}^{-4}$ presented by Ulbricht (1977). We test the effect of decreasing values of $N_{0}$ for rain and hail by an order of magnitude. This decrease is chosen to be large enough to roughly account for the maximum potential variability in $N_{0}$. The result is a $55 \%$ increase in $\sigma_{\mathrm{TV}_{\text {rain }}}^{2}$ and a $39 \%$ increase in $\sigma_{\mathrm{TV}_{\text {hail }}}^{2}$. Such a large increase in $\sigma_{\mathrm{TV} \text { rain }}^{2}$ is unlikely given the confidence in our selection of $N_{0_{\text {rain }}}$ (Marshall and Palmer, 1948). However, the corresponding increase for $\sigma_{\mathrm{TV}}^{2}$ hail is more likely realised given the stated uncertainty in $N_{0_{\text {hail }}}$. Such an increase would imply that $\sigma_{\mathrm{TV}}^{2}(60 \mathrm{dBZ})<\sigma_{\text {neil }}^{2}$ only if observed at $\theta_{\mathrm{el}}<9.8^{\circ}$. By instead increasing values of $N_{0}$ by an order of magnitude (not shown), we see a reduction in $\sigma_{\mathrm{TV} \text { hail }}^{2}$ and $\sigma_{\mathrm{TV}_{\text {rain }}}^{2}$ of $36 \%$ and $28 \%$, respectively.

A final source of uncertainty lies with the selected velocity-diameter relationship for hail, $V_{\text {hail }}(D)$. There is a broader diversity in these relationships in the literature than for rain; we assume 


\section{Observing turbulence in convective clouds}

386

387

388

389

390

391

392

393

394

395

396

397

398

399

400

401

402

403

404

405

406

407

the $V_{\text {rain }}(D)$ power law provided by Atlas and Ulbricht (1977) to be accurate. Figure 1b compares $\sigma_{\mathrm{TV}}^{2}$ hail from (13) derived using $V_{\text {hail }}(D)$ from Cheng and English (1982), Ulbricht (1977), and Pruppacher and Klett (1978). As the $V_{\text {hail }}(D)$ proposed by Ulbricht (1977) involves the same exponent $(q=0.5)$ as that used for $(13)$, the resulting effect is a $29 \%$ increase in $\sigma_{T V_{\text {hail }}}^{2}$ for all reflectivity owing to the different values of $p$. The $V_{\text {hail }}(D)$ relationship from Pruppacher and Klett (1978) however, involves $q=0.8$. This leads to a change in exponent in (13), causing a decrease in $\sigma_{\mathrm{TV}}^{2}(Z<40 \mathrm{dBZ})$ and an increase for $\sigma_{\mathrm{TV}}^{2}(Z>40 \mathrm{dBZ}) . \sigma_{\mathrm{TV}}^{2}\left(\mathrm{~V}_{\text {hail }}(60 \mathrm{dBZ})\right.$ is increased by $43 \%$. Figure $1 \mathrm{~b}$ suggests that the selection of $V_{\text {hail }}(D)$ can have a substantial and varied effect on $\sigma_{\mathrm{TV}}^{2}$ hail which somewhat limits how precisely we can state the conditions that allow us to neglect $\sigma_{\mathrm{TV}}^{2}$ hail .

\section{Spectral variance due to antenna rotation, $\sigma_{\alpha}^{2}$ and hydrometeor oscillations, $\sigma_{0}^{2}$.}

The movements of the radar antenna while scanning will broaden the Doppler spectrum. Assuming a constant antenna scan rate $\alpha$, in $\operatorname{rad~s}^{-1}$, the spectral variance contribution due to antenna rotation is provided by Doviak and Zrnic (1984),

$$
\sigma_{\alpha}^{2}=\left(\frac{\alpha \lambda \cos \theta_{\mathrm{el}} \sqrt{\ln (2)}}{2 \pi \theta_{1}}\right)^{2}
$$

where $\lambda$ is the wavelength of the radar in metres, $\theta_{\mathrm{el}}$ is the elevation angle from the surface, and $\theta_{1}$ is the one-way half-power beam width in radians.

For CAMRa, $\lambda=0.0975 \mathrm{~m}$ and $\theta_{1}=5 \times 10^{-3} \mathrm{rad}$. During DYMECS, RHI and PPI observations were made using scan speeds of $\alpha_{\mathrm{RHI}}=7 \times 10^{-3} \mathrm{rad} \mathrm{s}^{-1}$ and $\alpha_{\mathrm{PPI}}=35 \times$ $10^{-3} \mathrm{rad} \mathrm{s}^{-1}$. The contribution from $\sigma_{\alpha}^{2}$ is largest when scanning horizontally $\left(\cos \left(\theta_{\mathrm{el}}=0\right)=1\right)$; in this case $\sigma_{\alpha}^{2}<0.01 \mathrm{~m}^{2} \mathrm{~s}^{-2}$ for both RHI and PPI observations, making a negligible $\left(\sigma_{\alpha}^{2}<\sigma_{\text {neg }}^{2}\right)$ contribution to $\sigma_{v}^{2}$. Observations collected at non-zero elevations (up to $15^{\circ}$ in DYMECS) would 


\section{Observing turbulence in convective clouds}

only reduce the value of $\sigma_{\alpha}^{2}$. Equation (14) can be used simply to determine the contribution of $\sigma_{\alpha}^{2}$ for radars with much faster scanning speeds.

The oscillation of hydrometeors can contribute to $\sigma_{v}^{2}$, and has been speculated in Zrnic and Doviak (1989) to lead to over-estimation of $\varepsilon$. They find that $\sigma_{0}^{2}$ decreases with rain-rate, and generally does not increase above $0.25 \mathrm{~m}^{2} \mathrm{~s}^{-2}$, so we neglect these contributions.

\section{Spectral broadening due to shear of the radial wind, $\sigma_{S}^{2}$}

\subsection{Spectral variance equations for shear}

Since we are justified in neglecting $\sigma_{\mathrm{TV}}^{2}, \sigma_{\alpha}^{2}$ and $\sigma_{\mathrm{o}}^{2}$ in (1) for DYMECS observations, we are left with Doppler variance contributions from shear and turbulence. We can derive the turbulent contribution from,

$$
\sigma_{\mathrm{t}}^{2}=\sigma_{v}^{2}-\sigma_{s}^{2}
$$

for use in (2a) and (2b) to calculate $\varepsilon$.

In (15), $\sigma_{s}^{2}$ represents the sum of Doppler variance contributions from the shear of the radial wind in the elevation $\theta$, azimuthal (transverse across the beam) $\varphi$, and radial $r$, directions. Similar to $\sigma_{v}^{2}$, $\sigma_{s}^{2}$ can be decomposed into a sum of statistically independent variance contributions from shear in each direction.

$$
\sigma_{s}^{2}=\sigma_{s \theta}^{2}+\sigma_{s \varphi}^{2}+\sigma_{s r}^{2}
$$

Various equations have been used in past literature to calculate $\sigma_{s \theta}^{2}$ and $\sigma_{s \varphi}^{2}$ that are not mutually consistent (e.g. Chapman and Browning, 2001). In Appendix S1, we provide a derivation of these equations that produces results in agreement with those stated in Doviak and Zrnic (1984). An expression for $\sigma_{s r}^{2}$ is also taken from Doviak and Zrnic (1984), assuming a rectangular transmitted pulse. 


\section{Observing turbulence in convective clouds}

$$
\sigma_{s \theta}^{2}=\frac{\left(\left|S_{\theta}\right| R \theta_{1}\right)^{2}}{16 \ln 2}
$$

$$
\sigma_{s \varphi}^{2}=\frac{\left(\left|S_{\varphi}\right| R \theta_{1}\right)^{2}}{16 \ln 2}
$$

$$
\sigma_{s r}^{2}=\left(\frac{0.35\left|S_{r}\right| c \tau}{2}\right)^{2}
$$

Where $R$ is the radial distance from the radar in metres, $c$ is the speed of light in $\mathrm{m} \mathrm{s}^{-1}$, and $\tau$ is the pulse duration in seconds (for CAMRa, $\tau=0.5 \mu \mathrm{s}$ ). $\left|S_{\theta}\right|,\left|S_{\varphi}\right|$ and $\left|S_{r}\right|$ are shear magnitudes in $\mathrm{s}^{-1}$, calculated from the mean Doppler velocity field. In (17) - (19), velocity and reflectivity gradients are assumed to be linear across $V_{6}$. Equations (17) and (18) differ only in the shear involved, as the beam profiles in the $\theta$ and $\varphi$ dimensions are the same.

In application to CAMRa, the variability of $\sigma_{s(\theta, \varphi, r)}^{2}$ with $|S|$ and $R$ is illustrated in Figure 1c. For $\left|S_{r}\right|$ in the range of 0 to $0.02 \mathrm{~s}^{-1}, \sigma_{s r}^{2}$ increases with $\left|S_{r}\right|^{2}$ from 0 to $0.28 \mathrm{~m}^{2} \mathrm{~s}^{-2}$. As the pulse length is constant, $\sigma_{s r}^{2}$ does not vary with range. If $\left|S_{r}\right|<0.027 \mathrm{~s}^{-1}$ then $\sigma_{s r}^{2}<\sigma_{\text {neg, }}^{2}$, indicating that for our observations, $\sigma_{s r}^{2}$ is negligibly small except in cases of extreme shear. However, although $\sigma_{s r}^{2}$ is likely to be small, our chosen method of calculating shear (Section 5.3) permits direct measurement of $\left|S_{r}\right|$ to be made simply. We therefore include contributions from $\sigma_{s r}^{2}$ in $\sigma_{s}^{2}$.

At $30 \mathrm{~km}$ range, for $\left|S_{\theta, \varphi}\right|$ in the range of $0-0.02 \mathrm{~s}^{-1}, \sigma_{s(\theta, \varphi)}^{2}$ increases from $0-0.75 \mathrm{~m}^{2} \mathrm{~s}^{-2}$. At $150 \mathrm{~km}$ range, this increase is to $18.7 \mathrm{~m}^{2} \mathrm{~s}^{-2}$ when $\left|S_{\theta, \varphi}\right|$ is $0.02 \mathrm{~s}^{-1}$. This suggests that, even at the minimum range of $30 \mathrm{~km}$, if $\left|S_{\theta, \varphi}\right|>0.016 \mathrm{~s}^{-1}$, then $\sigma_{s(\theta, \varphi)}^{2}$ is always greater than $\sigma_{\text {neg }}^{2}$ for our data. Given that shears of this magnitude are quite possible (especially in the elevation direction), $\sigma_{s(\theta, \varphi)}^{2}$ will need to be considered for all of our observations. The high resolution of CAMRa means that radial velocity shear measured over small distances often result in negligible (less than $0.5 \mathrm{~m}^{2} \mathrm{~s}^{-2}$ ) contributions to $\sigma_{v}^{2}$, however, as illustrated, this is not true for shear of sufficient values. To ensure 


\section{Observing turbulence in convective clouds}

accuracy in point-to-point values of $\varepsilon$, and consistency in application across full RHI scans, we measure and remove $\sigma_{s}^{2}$ at each point in our data. In Section 6.2, we provide and discuss an example retrieval for a shower cloud (Figure 8) in which $\sigma_{s}^{2}$ exceeds $\sigma_{\text {neg }}^{2}$ quite widely and represents a significant proportion of $\sigma_{\mathrm{t}}^{2}$. This example is used to highlight the potential for significant overestimation of $\varepsilon$ in our cases if shear corrections are neglected.

\subsection{Separation of shear and turbulence - theory}

The separation of shear and turbulence is a significant challenge. However, methods to make this distinction are guided by the framework employed to derive $\varepsilon$ from $\sigma_{v}^{2}$ summarised in Section 2.2. The calculation of $\sigma_{s}^{2}$ is necessary to remove velocity variance contributions to $\sigma_{v}^{2}$ from outside the range of scales sampled by the radar. The scale over which to calculate shear (hereafter referred to as $\left.\Lambda_{s}\right)$ in $(17)$ - (19), should ideally be equal to the largest scale of the inertial sub-range, $\Lambda_{0}$. However, $\Lambda_{s}$ should be strictly no larger than $\Lambda_{0}$, otherwise the inclusion in $\sigma_{\mathrm{t}}^{2}$ of variance from outside the inertial sub-range will lead to an over-estimation of $\varepsilon$.

Without the means to routinely estimate $\Lambda_{0}$ for each of the convective storm observations collected in DYMECS, we refer to past literature. Past studies have utilised Doppler spatial spectra and aircraft measurements to estimate $\Lambda_{0}$ in individual convective clouds (Battan, 1975; Knupp and Cotton, 1982; Brewster and Zrnic, 1986). They found that $\Lambda_{0}$ can be as large as $1.5-3 \mathrm{~km}$. These estimates were made in severe thunderstorms/hailstorms with strong, large-scale circulations. In comparison, the convective storms constituting the DYMECS observations are generally much weaker, limiting how applicable these values are to our cases. We assume $\Lambda_{0}$ scales with the size of the largest eddy-generating mechanisms in a convective cloud, i.e. the main updraft circulation. If this circulation is shallow, we expect $\Lambda_{0}$ to be small as the downscale cascade to isotropic turbulence begins at a smaller eddy scale. As updraft heights on DYMECS case days generally ranged from 3 $8 \mathrm{~km}$ (Nicol et al., 2015), we assume $\Lambda_{0} \sim 1 \mathrm{~km}$ for this application. Chapman and Browning (2001) found a factor two change in $\Lambda_{0}$ to have very little effect on their resulting values of $\varepsilon$. However, this 


\section{Observing turbulence in convective clouds}

477

478

479

480

481

482

483

484

involved assuming a $\Lambda_{0}$ of only $200 \mathrm{~m}$ for shallow shear layers, so we test the sensitivity of our retrieved $\varepsilon$ to the value of $\Lambda_{s}$, summarised in Section 5.5.

\subsection{Separation of shear and turbulence - linear velocity surface approach}

The application of methods to distinguish $\sigma_{s}^{2}$ from $\sigma_{\mathrm{t}}^{2}$ will depend on the relationship between the spatial resolution of the radar and $\Lambda_{0}$. As long as the largest dimension of $V_{6}$ is less than $\Lambda_{0}, \sigma_{\mathrm{t}}^{2}$ can be used to estimate $\varepsilon$ (Frisch and Clifford, 1974). As this is generally the case for a radar of CAMRa's resolution, scanning deep convective clouds, $\sigma_{s}^{2}$ can then be determined from radial velocity shear calculated over enough contiguous $V_{6}$ volumes to constitute a spatial scale of $\Lambda_{0}$.

To evaluate shear over a constant spatial scale in data with polar co-ordinates is not straightforward. With two-dimensional radar data, the most effective way to achieve this is to use leastsquares regression to fit a velocity surface to Doppler velocity data. A suitable framework for this velocity surface is taken from Neter and Wasserman (1974), and has been applied in previous $\varepsilon$ retrieval studies (Istok and Doviak 1986, Meischner et al., 2001). When applied to RHIs, the velocity surface is given by

$$
V_{i}=V_{0}+S_{\theta} l_{\theta_{i}}+S_{r} l_{r_{i}}+E_{i}
$$

where

$$
l_{\theta_{i}}=R_{0}\left(\theta_{i}-\theta_{0}\right) \quad ; \quad l_{r_{i}}=R_{i}-R_{0}
$$

The range from the radar is given by $R, \theta$ is the elevation angle in radians, and $\left(\theta_{0}, R_{0}\right)$ is the centre point of the surface. $V_{i}$ is the radial velocity at the point $\left(\theta_{i}, R_{i}\right)$, and $E_{i}$ is the velocity difference between the data and the surface. $V_{0}$ is the estimated central point velocity and $S_{\theta}$ and $S_{r}$ are linear elevation and radial shears, respectively. $l_{\theta_{i}}$ and $l_{r_{i}}$ are the elevation and radial distances between $V_{i}$ and $V_{0}$. 


\section{Observing turbulence in convective clouds}

The parameters $V_{0}, S_{\theta}$ and $S_{r}$ are determined from the matrix operation,

$$
\left[\begin{array}{l}
V_{0} \\
S_{\theta} \\
S_{r}
\end{array}\right]=\left[\begin{array}{ccc}
n & \sum l_{\theta_{i}} & \sum l_{r_{i}} \\
\sum l_{\theta_{i}} & \sum l_{\theta_{i}}^{2} & \sum l_{r_{i}} l_{\theta_{i}} \\
\sum l_{r_{i}} & \sum l_{\theta_{i}} l_{r_{i}} & \sum l_{r_{i}}^{2}
\end{array}\right]^{-1}\left[\begin{array}{c}
\sum V_{i} \\
\sum V_{i} l_{\theta_{i}} \\
\sum V_{i} l_{r_{i}}
\end{array}\right]
$$

\section{Centred to best approximation on a chosen Doppler velocity point, neighbouring data points are} used to constitute (as closely as is possible) a $\Lambda_{s}-$ by $-\Lambda_{s}$ grid of data, $G$. Using velocities from $G$, (21) is used to compute linear shears $S_{\theta}$ and $S_{r}$, which are attributed back to the data point at the centre of $G$. By completing this process for all points in a scan, we obtain fields of $S_{\theta}$ and $S_{r}$, calculated over a fixed scale. If $\Lambda_{s}=\Lambda_{0}, S_{\theta}$ and $S_{r}$ are representative of large eddies and/or velocity gradients in the ordered background flow. As a result, they can be used in (17) and (19) to determine $\sigma_{s \theta}^{2}$ and $\sigma_{s r}^{2}$

In applying (20) to our observations, we find that data located less than $\sim \frac{\Lambda_{s}}{2}$ from the edge of observed clouds will be lost in surface fitting. The grid $G$ will only be partially filled with data for those $V$ located on the periphery of reflectivity echoes, meaning (21) cannot be performed. The degree of data loss therefore increases with the value of $\Lambda_{s}$. As we can only account for $\sigma_{s}^{2}$ where shear can be measured, this data loss is imposed on retrieved fields of $\varepsilon$. Consequently, this limits our ability to investigate values of $\varepsilon$ associated with entrainment processes near cloud edges. However, turbulence associated with entrainment into updrafts can still be retrieved in cases where updrafts are further than $\sim \frac{\Lambda_{s}}{2}$ from the edge of the radar echo (e.g. Figure 6c and 6f). Although we lose peripheral data, we benefit from the removal of noise in low reflectivity areas around cloud edges, which can develop large values of $\sigma_{v}^{2}$. The 300-m range resolution of our observations restricts values of $\Lambda_{s}$ to multiples of $300 \mathrm{~m}$ in order to include whole radial cells, and a minimum of $600 \mathrm{~m}$ to include at least two radial cells for the calculation of shear. Under these restrictions, assuming $\Lambda_{0} \sim$ $1 \mathrm{~km}$, we select $\Lambda_{s}=900 \mathrm{~m}$ for our observations. 


\section{Observing turbulence in convective clouds}

523

524

525

526

527

528

529

530

531

532

533

\subsection{Variance from azimuthal shear, $\sigma_{s \varphi}^{2}$}

When RHI or PPI scans are performed, the radial velocity field is observed in two dimensions, the radial direction and the scanning direction. However, these fields include data from threedimensional sample volumes. In terms of RHIs, Doppler variance from azimuthal shear, $\sigma_{s \varphi}^{2}$ contributes to $\sigma_{v}^{2}$, but we are unable to directly estimate it due to scanning in the elevation direction. Unless an adjacent RHI is performed, separated from the first by an angular distance comparable to the width of the beam, $S_{\varphi}$ cannot be determined directly. As shown in Section 5.1, variance contributions from $\sigma_{s \varphi}^{2}$ cannot be ignored in our observations. To account for $\sigma_{s \varphi}^{2}$ in circumstances where it cannot be measured directly, we investigate statistical relationships between $\left|S_{\varphi}\right|$ and $\left|S_{r}\right|$ derived from PPI radar observations.

PPI scans were performed alongside RHIs scans on DYMECS case days. Doppler velocity fields from PPI scans can be differentiated in the radial and azimuthal directions to determine fields of $\left|S_{\varphi}\right|$ and $\left|S_{r}\right|$. By collecting many co-located pairs of $\left|S_{\varphi}\right|$ and $\left|S_{r}\right|$ from these fields, we attempt to parametrise $\left|S_{\varphi}\right|$ as a function of $\left|S_{r}\right|$. Using the result, $\left|S_{r}\right|$ found in RHIs can be used to estimate $\left|S_{\varphi}\right|$, and its uncertainty, allowing us to account for all components of $\sigma_{s}^{2}$ in RHI scans.

In order for relationships derived between $\left|S_{\varphi}\right|$ and $\left|S_{r}\right|$ to be of most benefit, we must impose they are calculated over a mutual spatial scale, consistent with that used to calculate $\left|S_{\theta}\right|$ and $\left|S_{r}\right|$ in RHIs, i.e. $\Lambda_{s}=900 \mathrm{~m}$. To achieve this, we use a version of (20) tailored to PPI scans, where $S_{\theta} l_{\theta_{i}}$ is replaced by $S_{\varphi} l_{\varphi_{i}}$, and $l_{\varphi_{i}}$ is the azimuthal distance between $V_{i}$ and $V_{0}$. By generating $\left|S_{\varphi}\right|$ and $\left|S_{r}\right|$ values for all $V_{6}$ across many PPIs, we could build a dataset consisting of co-located values of $\left|S_{\varphi}\right|$ and $\left|S_{r}\right|$ for statistical assessment.

Figure 2 shows the independent distributions of approximately $10^{6}$ values of $S_{\varphi}$ and $S_{r}$ sourced from 31 PPIs taken on 20 April 2012 at varying elevations. $S_{\varphi}$ and $S_{r}$ are both approximately normally distributed. The combined two-dimensional distribution of $S_{\varphi}$ and $S_{r}$ is circular Gaussian, 


\section{Observing turbulence in convective clouds}

547

approximately centred on $S_{r}=S_{\varphi}=0$. Once the magnitude of the values in the combined distribution is taken, which is the quantity relevant to $\sigma_{s}^{2},\left|S_{r}\right|$ is divided into contiguous intervals of width $1 \times 10^{-4} \mathrm{~s}^{-1}$. For each of these, we extract the associated dataset of $\left|S_{\varphi}\right|$, and generate its probability density function (PDF). Figure 3 demonstrates that the resulting PDFs are very well approximated by the gamma distribution, given for a random variable $x$, by (22).

$$
\gamma(x \mid k, l)=\frac{x^{k-1} e^{-\frac{x}{l}}}{\Gamma(k) l^{k}}
$$

Using (22), we can accurately simulate the change in the distribution of $\left|S_{\varphi}\right|$ with $\left|S_{r}\right|$. For each $\left|S_{r}\right|$ interval, we extract the gamma distribution parameters $k$ (shape) and $l$ (scale) from the corresponding distribution of $\left|S_{\varphi}\right|$. By numerically fitting functions to relationships between $(k, l)$ and $\left|S_{r}\right|$, we define $k$ and $l$ in terms of $\left|S_{r}\right|$,

$$
\begin{gathered}
k= \begin{cases}\left|S_{r}\right|\left(A_{1}\left|S_{r}\right|+A_{2}\right)+A_{3}, & \text { if }\left|S_{r}\right| \leq 0.0017 \mathrm{~s}^{-1} \\
B_{1}\left|S_{r}\right|+B_{2}, & \text { otherwise }\end{cases} \\
\quad l=C_{1}\left|S_{r}\right|+C_{2}
\end{gathered}
$$

where coefficient values are provided in Table 2. For a given value of $\left|S_{r}\right|$, we use (23) and (24) to produce a PDF of $\left|S_{\varphi}\right|$, and derive our estimate of $\left|S_{\varphi}\right|$ as the mean of this distribution. For a gamma distribution, the mean is simply the product of $k$ and $l$.

Figure 4 shows the change in median and inter-quartile range (IQR) percentiles of $\left|S_{\varphi}\right|$ with $\left|S_{r}\right|$. Distributions of $\left|S_{\varphi}\right|$ get broader with $\left|S_{r}\right|$. As a result, the size of the IQR, which provides a confidence interval for $\left|S_{\varphi}\right|$, increases with $\left|S_{r}\right|$. The median values of $\left|S_{\varphi}\right|$ increase with $\left|S_{r}\right|$ according to (25) which was obtained by least-squares fitting a quadratic function to the median curve in Figure 4. Mean values of $\left|S_{\varphi}\right|$ also increase with $\left|S_{r}\right|$, with values approximately $25 \%$ larger than the median.

$$
\left|S_{\varphi}\right|_{\text {med }}=\left|S_{r}\right|\left(D_{1}\left|S_{r}\right|+\mathrm{D}_{2}\right)+D_{3}
$$




\section{Observing turbulence in convective clouds}

569

570

571

572

573

574

575

576

577

578

579

580

581

582

583

584

585

586

587

588

589

590

591

where coefficient values are provided in Table 2.

Using (23) - (25), $\left|S_{\varphi}\right|$, and an estimate of its uncertainty, can be determined from $\left|S_{r}\right|$ alone. $\left|S_{\varphi}\right|$ is then used in (18) to calculate its variance contribution, $\sigma_{s \varphi}^{2}$. We can now account for all components of $\sigma_{s}^{2}$ in (16), subtract $\sigma_{s}^{2}$ from $\sigma_{v}^{2}$ to find $\sigma_{\mathrm{t}}^{2}$ using (15), and use $\sigma_{\mathrm{t}}^{2}$ in (2a) and (2b) to determine $\varepsilon$.

\subsection{Sensitivity of $\varepsilon$ to $\Lambda_{s}$}

To perform this sensitivity test, $\varepsilon$ is determined in RHI scans of convective clouds using different values of $\Lambda_{s}$ in methods to calculate $\sigma_{s}^{2}$. We use 44 RHI scans performed on 25 August 2012 which provide $3.5 \times 10^{5}$ comparable data points for each $\Lambda_{s}$ applied. For all scans, we retrieve $\varepsilon\left(\Lambda_{s}\right)$ where $\Lambda_{s}$ is $600 \mathrm{~m}, 900 \mathrm{~m}, 1500 \mathrm{~m}, 2100 \mathrm{~m}$, and $2700 \mathrm{~m}$. As described in Section 5.3, the degree of peripheral data loss in velocity surface fitting increases with $\Lambda_{s}$. To ensure that we are comparing the same data across different $\Lambda_{s}$, the degree of data loss seen when $\Lambda_{s}=2700 \mathrm{~m}$ has been imposed on all other fields of $\varepsilon$ for each scan.

Figure 5 displays the PDFs of $\varepsilon\left(\Lambda_{s}\right)$ using the combined data from all RHIs. It shows that the distribution of $\varepsilon$ is largely insensitive to $\Lambda_{s}$, though there is a small increase in the likelihood of low values of $\varepsilon$ (less than $0.01 \mathrm{~m}^{2} \mathrm{~s}^{-3}$ ) with decreased $\Lambda_{s}$. When calculating shear over a smaller $\Lambda_{s}$, the shear magnitude, and therefore $\sigma_{s}^{2}$, is likely to be higher. This means we remove more of $\sigma_{v}^{2}$ due to shear, and subsequently derive a lower $\varepsilon$, with the converse true if $\Lambda_{s}$ is large. As the change in PDFs of $\varepsilon$ is small in Figure 5, we can make rough estimations of $\Lambda_{0}$ (and therefore $\Lambda_{S}$ ) in the absence of direction measurements, without incurring large errors in $\varepsilon$.

\section{Dissipation rate statistics in DYMECS observations}

\subsection{DYMECS case studies}




\section{Observing turbulence in convective clouds}

592

By applying the methods detailed in Section 2 - 5 across many radar scans, we have performed a statistical assessment of $\varepsilon$ in convective storms. We use two contrasting DYMECS days in 2012 as case studies; 20 April (hereafter the "shower" case) and 25 August (hereafter "deep convection" case). In the shower case, low pressure was situated on the east coast of the UK. Convective showers initiated over southern England in the late morning hours, and drifted north-eastwards through the day. In the deep convection case, low pressure was situated over the Irish Sea. Convective storms were more intense and widespread across southern England, with thunderstorms widely reported in the afternoon (Hanley et al., 2015). Radar observations were collected using CAMRa in both cases, using a scanning algorithm that prioritised more active convective cells, guided in real time by Met Office network radar observations (Stein et al., 2014). As this scanning strategy involved sequential scans of the same cells, a subset of these observations has been taken to include only independent convective storm RHI observations for analysis. This subset consists of 33 RHIs in the shower case, and 44 RHIs for deep convection, however, owing to the 200-km range of CAMRa, multiple convective storms were often present in single RHI scans. In the shower case, these observations show that convection grew to $6 \mathrm{~km}$ in height, with updraft vertical velocities $w$, typically ranging from $1-4 \mathrm{~m} \mathrm{~s}^{-1}$. In the deep convection case, convection grew to $10 \mathrm{~km}$ with typical $w$ ranging from $2-8 \mathrm{~m} \mathrm{~s}^{-1}$ (Nicol et al., 2015).

\subsection{Example \& retrievals for convective showers and deep convection}

Figures 6 and 7 display examples of retrieved $\varepsilon$ for individual convective clouds on the shower and deep convection case days, respectively. These examples have been selected to reflect the typical convective storms observed on each day. Figure 6 depicts a convective shower of $6 \mathrm{~km}$ height, with a diffuse updraft region where $w$ is $1-3 \mathrm{~m} \mathrm{~s}^{-1}$ (Figure 6c), with a region of strong divergence present in the Doppler velocity above the updraft (Figure 6b). As shown in Figure 6f, $\varepsilon$ typically ranges from $0.01-0.08 \mathrm{~m}^{2} \mathrm{~s}^{-3}$ with the largest values found within the vicinity of the main updraft. 


\section{Observing turbulence in convective clouds}

The example of deep convection displayed in Figure 7 has a depth of $10 \mathrm{~km}$, and shows multi-cell characteristics with numerous updraft-downdraft circulations present in Figure $7 \mathrm{c}$. The dominant updraft ( $34 \mathrm{~km}$ from the radar) is narrower and much stronger than for the shower case, with $w$ ranging from $8-12 \mathrm{~m} \mathrm{~s}^{-1}$. Divergence is again apparent in the Doppler velocity towards the upper levels of the cloud (Figure $7 \mathrm{~b}$ ). Figure $7 \mathrm{f}$ indicates that turbulence is more intense and widespread than for the shower case, with values of $\varepsilon$ typically ranging from $0.03-0.3 \mathrm{~m}^{2} \mathrm{~s}^{-3}$. These values are again associated with the main updraft, with the most intense turbulence $\left(\varepsilon>0.3 \mathrm{~m}^{2} \mathrm{~s}^{-3}\right)$ found towards the top of the cloud, above the updraft.

In many of the cloud cases that were examined to derive the statistics of $\varepsilon$ presented later in this section, values of $\sigma_{s}^{2}$ were small compared to $\sigma_{\mathrm{t}}^{2}$ and largely remained below $\sigma_{\text {neg. }}^{2}$. The retrievals presented in Figures 6 and 7 provide examples of this; values of $\sigma_{s}^{2}>\sigma_{\text {neg }}^{2}$ were absent in the shower cloud and were restricted to a small cluster of 36 pulse volumes in the deep cloud case. This region is evident in Figure 7e, located from $7.5-8 \mathrm{~km}$ in height at an approximate range of $33-34 \mathrm{~km}$. Although $\sigma_{s}^{2}$ was significant in this region, values remained less than $15 \%$ of $\sigma_{\mathrm{t}}^{2}$ suggesting that $\sigma_{s}^{2}$ could have been neglected in these two cloud examples without significantly over-estimating $\varepsilon$.

Shear corrections are, however, not negligible for all cloud cases considered, especially for those located further from the radar. According to (17) and (18), variances from azimuthal and elevation shear components increase with range squared. Figure 8 presents the ratio of $\sigma_{s}^{2}$ to $\sigma_{\mathrm{t}}^{2}$ in an example cloud observed between $90-115 \mathrm{~km}$ from the radar on 20 April 2012. Within the region of $\sigma_{s}^{2}>$ $\sigma_{\text {neg }}^{2}$ (black contour) values of $\sigma_{s}^{2}$ vary between $30 \%-70 \%$ of $\sigma_{\mathrm{t}}^{2}$. Neglecting $\sigma_{s}^{2}$ in this region would result in the over-estimation of mean $\varepsilon$ by $52 \%$. Given that clouds were commonly observed $100 \mathrm{~km}$ (or further) from the radar, Figure 8 provides an example of the requirement to remove $\sigma_{s}^{2}$ in our data to ensure accurate retrievals of $\varepsilon$.

By inspecting many retrievals of $\varepsilon$ in convective clouds across two contrasting days of convection, the rest of this section is focused on relating $\varepsilon$ to convective storm characteristics in a statistical sense. 


\section{Observing turbulence in convective clouds}

642

643

644

645

646

647

648

649

650

651

652

653

654

655

656

657

658

659

660

661

662

663

664

665

\subsection{Vertical distribution of $\varepsilon$ in convective clouds}

Statistics have been collected for $\varepsilon$ in vertical layers of 1-km depth from the subsets of RHI observations described in Section 6.1. Using this approach, we can assess the vertical distribution of $\varepsilon$ in convective clouds (where $Z>-20 \mathrm{dBZ}$ ), and see how this differs for showers and deep convection.

Figure 9 shows how the median, and $25^{\text {th }}, 75^{\text {th }}$ and $95^{\text {th }}$ percentiles of $\varepsilon$ change with height in the cloud; $0-6 \mathrm{~km}$ for showers, $0-10 \mathrm{~km}$ for deep convection. For showers and deep convection, the $95^{\text {th }}$ percentiles of $\varepsilon$ (hereafter $\varepsilon_{95}$ ) from $0-1 \mathrm{~km}$ are approximately the same, at $\sim 0.025 \mathrm{~m}^{2} \mathrm{~s}^{-3}$. For the shower case, this remains approximately constant with height, varying between $0.02-0.03$ $\mathrm{m}^{2} \mathrm{~s}^{-3}$ and peaking at a height of $5 \mathrm{~km}$. For deep convection, $\varepsilon_{95}$ increases significantly with height, becoming twice as large as for the shower case at $6 \mathrm{~km}$ height $\left(0.05 \mathrm{~m}^{2} \mathrm{~s}^{-3}\right)$, and increasing to 0.1 $\mathrm{m}^{2} \mathrm{~s}^{-3}$ at $10 \mathrm{~km}$ height. The median $\varepsilon$ is an approximately constant $0.01 \mathrm{~m}^{2} \mathrm{~s}^{-3}$ throughout the 6$\mathrm{km}$ depth of the shower cases. For the same depth of deep convective cloud, the median is almost identical to the shower case, and then increases from $0.01-0.03 \mathrm{~m}^{2} \mathrm{~s}^{-3}$ from $6-10 \mathrm{~km}$.

The $25^{\text {th }}$ and $75^{\text {th }}$ percentiles of $\varepsilon$ follow a very similar pattern to this, indicating that from $0-6$ $\mathrm{km}$ the average intensity of turbulence is very similar for both cases. The reason for this is highlighted by Figure $6 \mathrm{f}$ and $7 \mathrm{f}$; turbulence is locally intense, but a large proportion of the cloud area is only weakly turbulent in both cases $\left(\varepsilon \leq 0.01 \mathrm{~m}^{2} \mathrm{~s}^{-3}\right)$. This was often the case throughout observations on both days which serves to explain why the bulk of $\varepsilon$ values are so similar. Where the cloud is turbulent however, values of $\varepsilon$ are much larger in deep convection with much stronger circulations, which is reflected in the notable difference in $\varepsilon_{95}$ between the two cases. 


\section{Observing turbulence in convective clouds}

666

667

668

669

670

671

672

673

674

675

676

677

678

679

680

681

682

683

684

685

686

687

688

689

690

As we are most interested in the turbulent regions of individual convective clouds, and turbulence in observations tends to be associated with convective updrafts (see Figures 6 and 7), we refine this investigation to $\varepsilon$ located only in convective updraft regions.

The following method has been selected in part to ensure it can be applied identically to numerical model data in future investigations. To detect coherent updraft regions, a flood-fill algorithm is applied to vertical cross-sections of $w$ (T. Stein, personal communication), to automatically detect contiguous regions with $w$ above specified thresholds, and record their co-ordinates. By taking the four spatial extremes of these co-ordinates, a box is drawn around an updraft - defined as an updraft region. Updrafts are often irregular in shape in observations, so this approach includes some data surrounding the updraft in the defined region. As a result, we benefit from including some information about turbulence associated with an updraft, without it having to be co-located with specific values of $w$.

Once updraft regions are defined, they are filtered by width and depth to avoid the inclusion of very small, insignificant updrafts that are detected by the algorithm. For the shower case, we used a minimum threshold $w$ of $1 \mathrm{~m} \mathrm{~s}^{-1}$, and a minimum depth of $2 \mathrm{~km}$. For the deep convection case, we used a minimum threshold $w$ of $1.5 \mathrm{~m} \mathrm{~s}^{-1}$, and a minimum depth of $3 \mathrm{~km}$. In both cases, a minimum updraft region width of $1.5 \mathrm{~km}$ was imposed. The lower thresholds for $w$ and depth used in the shower case were chosen due to the weaker, shallower updrafts observed on that day. Using this approach, 77 updraft regions were detected in the shower case, and 101 regions for deep convection. The coordinates of each region can then be super-imposed on fields of $\varepsilon$ for analysis.

Figure 10 displays scatter plots relating $\varepsilon_{95}$ for each updraft region to its (a) $95^{\text {th }}$ percentile of $w$ (hereafter $w_{95}$ ), (b) $95^{\text {th }}$ percentile of the magnitude of the horizontal gradient in $w,\left|\frac{d w}{d x}\right|_{95}$, (c) updraft width, and (d) updraft depth. In Figure 10a, we see that $\varepsilon_{95}$ has a significant $\left(\mathrm{p}<10^{-3}\right.$ ) positive correlation with $w_{95}$ for both showers $(r=0.425)$ and deep convection $(r=0.594)$. Correlations with $\left|\frac{d w}{d x}\right|_{95}$ (Figure 10b) are marginally stronger than with $w_{95}(r=0.517$ for showers, $\mathrm{r}=0.671$ 


\section{Observing turbulence in convective clouds}

691 for deep convection). This suggests that strong gradients in $w$ are more important in generating

692 turbulence than $w$ alone. Weaker positive correlations exist between $\varepsilon_{95}$ and the width and depth of 693 updrafts for both showers $(r=0.295$ for width, and $r=0.314$ for depth), and deep convection 694 ( $r=0.309$ for width, and $r=0.390$ for depth). This indicates that the intensity of turbulence is

695

696

697

698

699

700

701

702

703

704

705

706

707

708

709

710

711

712

713

714

not highly sensitive to the dimensions of the updraft. The consistency of correlations between the two cases, albeit with a smaller range in variable values for the shower case, suggests that these relationships may not be restricted to individual cases, or days of observation.

To produce Figure 11, all $\varepsilon$ values in an updraft region are added to a distribution based on $w_{95}$. By doing this, we can assess how the full distribution of $\varepsilon$ changes with $w_{95}$ in the two cloud types, instead of just the largest values. These distributions are displayed in the form of cumulative density functions (CDFs) for every $2 \mathrm{~m} \mathrm{~s}^{-1}$ interval in $w_{95}$. In both the showers and deep convection, a trend towards a lower probability of small $\varepsilon$, and a higher probability of large $\varepsilon$ is seen with $w_{95}$. In both cases, small values of $\varepsilon$ (less than $0.01 \mathrm{~m}^{2} \mathrm{~s}^{-3}$ ) are approximately twice as likely to appear in updrafts with $w_{95}<4 \mathrm{~m} \mathrm{~s}^{-1}$, than for those $w_{95}>4 \mathrm{~m} \mathrm{~s}^{-1}$. In the shower case, $\varepsilon$ larger than $0.05 \mathrm{~m}^{2} \mathrm{~s}^{-3}$ has a probability of less than 0.01 in all updraft regions $\left(w_{95}<6 \mathrm{~m} \mathrm{~s}^{-1}\right)$; whereas for the same $w_{95}$ intervals of deep convection the probability is as large as 0.12 . This indicates that stronger turbulence is more likely to be found in deep convective clouds than for showers of the same updraft strength. However, we see only a snapshot of information for each convective cloud; turbulent energy will take time to reach dissipation scales, in which time updrafts could have weakened considerably. The probability of large values of $\varepsilon$ (more than $0.1 \mathrm{~m}^{2} \mathrm{~s}^{-3}$ ) is 0 for the shower case, but as high as 0.05 for deep convection. When $w_{95}$ is $2-4 \mathrm{~m} \mathrm{~s}^{-1}$, the CDFs of $\varepsilon$ are very similar for both cases, further indicating that $\varepsilon$ may be a function of storm characteristics independent of case, or day of observation.

\section{Summary and Conclusions}




\section{Observing turbulence in convective clouds}

A comprehensive analysis of processes contributing to the width of the Doppler velocity spectrum has been performed, with the objective of developing a rigorous algorithm to estimate turbulence intensity expressed as a dissipation rate.

New equations to quantify the spectral broadening effect due to a distribution of hydrometeor fall speeds $\left(\sigma_{\mathrm{TV}}^{2}\right)$ have been presented for ice aggregates, raindrops and hail. We conclude that $\sigma_{\mathrm{TV}}^{2}$ isg always negligibly small, and $\sigma_{\mathrm{TV}}^{2}$ rain and $\sigma_{\mathrm{TV}}^{2}$ hail are negligible when observing at elevations lower than $13.9^{\circ}$ and $11.5^{\circ}$, respectively. We find that $\sigma_{\mathrm{TV}}^{2}$ can be larger than $8 \mathrm{~m}^{2} \mathrm{~s}^{-2}$ if scanning vertically through heavy rain or hail, and recommend avoiding high-elevation scanning when attempting to retrieve turbulence from the spectrum width.

Methods have been presented to remove contributions to $\sigma_{v}^{2}$ from shear over scales larger than those sampled by the radar. This was achieved by evaluating shear over a constant spatial scale $\left(\Lambda_{s}\right)$, using linear velocity surface fitting techniques as employed in past studies. Resulting values of $\varepsilon$ have been found to be insensitive to $\Lambda_{s}$. To permit the estimation of $\varepsilon$ from $\sigma_{\mathrm{t}}^{2}$, it is of key importance that the largest dimension of $V_{6}$ is lower than $\Lambda_{0}$.

To account for spectrum width contributions from shear in the azimuthal direction, we have derived a new equation for the median azimuthal shear as a function of radial shear alone. This can be used to account for 3-D shear broadening in 2-D radar scans, and can be used simply to further improve the accuracy of retrieved $\varepsilon$. After noting incorrect equations for the calculation of $\sigma_{s}^{2}$ in the literature, we conclude the correct equations are those derived in Appendix S1.

By applying the retrieval method across many observations on two contrasting DYMECS case days, we have produced statistics of $\varepsilon$ in convective clouds. Turbulence is generally much stronger in deep convective cloud $\left(0.03-0.3 \mathrm{~m}^{2} \mathrm{~s}^{-3}\right)$ than in shower cloud $\left(0.01-0.08 \mathrm{~m}^{2} \mathrm{~s}^{-3}\right)$. In both cases, the majority of cloud is generally weakly turbulent, with significant turbulence co-located with, but not limited to, areas of shear and buoyancy. Strong turbulence is more widespread towards the top of deep convective cloud, while vertical profiles of turbulence are approximately constant in 


\section{Observing turbulence in convective clouds}

740

shower cloud. In updraft regions, turbulence is strongly correlated with updraft strength, and there is evidence that gradients in the vertical velocity are more important in generating strong turbulence than the updraft velocity alone. Turbulence is only weakly correlated with the spatial dimensions of updrafts.

Our method has sourced, developed, and added to many decades of turbulence retrieval research to form the most comprehensive approach to date. Though we have ultimately applied the method to a specific radar and observational dataset, the considerations made in Sections $2-5$ are suitably general, forming a reliable framework for turbulence retrieval with other high-resolution radars capable of sampling inertial sub-range turbulence.

Following directly from this research, we have collected new observations of convective clouds with CAMRa, under an improved scanning strategy better suited to turbulence retrieval. By performing multiple RHI scans separated by small azimuthal distances across clouds, we aim to investigate the 3 -D structures of turbulence in convective storms.

We have also used the results of this investigation to evaluate the performance of the Smagorinsky-Lilly sub-grid scheme through direct comparisons with $\varepsilon$ in high-resolution NWP simulations of the observed cases. The degree to which our observations can be used more generally to evaluate turbulence characteristics in CPMs (without the need to simulate the observed cases) is not clear. However, at the very least, our observations can provide guidance for the typical characteristics of $\varepsilon$ in clouds for comparison with other high-resolution CPM simulations, given that $\varepsilon$ can be found as a diagnostic output from the turbulence parametrisation. To ultimately improve the versatility of our results, we aim to extend our observations to more diverse cloud cases to assess the degree to which our statistics are case-dependent.

\section{Acknowledgements}




\section{Observing turbulence in convective clouds}

This research was conducted with funding from the Natural Environment Research Council (NERC) under the SCENARIO programme. Acknowledgements go to Dr. John Nicol in providing vertical velocity retrievals for the case studies investigated, which have been invaluable to the analysis of turbulence in convective clouds. Further thanks go to staff at the Chilbolton Observatory for the technical support with data from CAMRa.

\section{Supporting information:}

\section{Appendix S1 - Derivation of spectral variance equations for shear}

\section{REFERENCES}

Albrecht BA, Fang M, Ghate VP. 2016. Exploring stratocumulus cloud-top entrainments processes and parameterizations by using Doppler cloud radar observations. J. Atmos. Sci., 54: 729-742.

Atlas D, Ulbricht CW. 1977. Path- and area-integrated rainfall measurement by microwave attenuation in the 1-3 cm band. J. Appl. Meteorol. 16: 1322-1331.

Battan LJ. 1975. Doppler radar observations of a hailstorm. J. Appl. Meteorol. 14: 98-108.

Blyth AM, Cooper AC, Jensen JB. 1988. A study of the source of entrained air in Montana Cumuli. J. Atmos. Sci. 45: 3944-3964.

Blyth AM. 1993. Entrainment in cumulus clouds. J. Appl. Meteorol. 32: 626-641.

Bouniol D, Illingworth AJ, Hogan RJ. 2003. Deriving turbulent kinetic energy dissipation rate within clouds using ground based $94 \mathrm{GHz}$ radar. Preprints, $31^{\text {st }}$ Conf. on Radar Meteorology, Seattle, WA, Amer. Meteor. Soc. 192-196.

Brewster KA, Zrnic DS. 1986. Comparison of eddy dissipation rates from spatial spectra of Doppler velocities and Doppler spectrum widths. J. Atmos. Oceanic Technol. 3: 440-552. 


\section{Observing turbulence in convective clouds}

Chapman D, Browning KA. 2001. Measurements of dissipation rate in frontal zones. Q. J. R. Meteorol. Soc. 127: 1939-1959.

Cheng L, English M. 1982. A relationship between hailstone concentration and size. J. Atmos. Sci. 40: 204-213.

Clark P, Roberts N, Lean H, Ballard SP, Charlton-Perez C. 2016. Convection-permitting models: a step-change in rainfall forecasting. Meteorol. Appl. 23: 165-181.

Cox GP. 1988. Modelling precipitation in frontal rainbands. Q. J. R. Meteorol. Soc. 114: 115-127.

Depue TK, Kennedy PC, Rutledge SA. 2007. Performance of the hail differential reflectivity $\left(H_{\mathrm{DR}}\right)$ polarimetric radar hail indicator. J. Appl. Meteorol. Climatol. 46: 1290-1301.

Doviak RJ, Zrnic DS. 1984. Doppler radar and weather observations. Academic Press.

Falkovich G, Fouxon A, Stepanov MG. 2002. Acceleration of rain initiation by cloud turbulence. Nature. 419: 151-154.

Fang M, Albrecht BA, Ghate VP, Kollias P. 2014. Turbulence in continental stratocumulus, part II: Eddy dissipation rates and large-eddy coherent structures. Bound.-Layer Meteor., 150: 361-380.

Frisch AS, Clifford SF. 1974. A Study of Convection Capped by a Stable Layer Using Doppler Radar and Acoustic Echo Sounders. J. Atmos. Sci. 31: 1622-1628.

Grover SN, Pruppacher HR. 1985. The effect of vertical turbulent fluctuations in the atmosphere on the collection of aerosol particles by cloud drops. J. Atmos. Sci. 42: 2305-2318.

Gunn KLS, Marshall RS. 1958. The distribution of size of aggregate snowflakes. J. Meteorol. Soc. 80: $522-545$.

Hanley KE, Plant RS, Stein THM, Hogan RJ, Nicol JC, Lean HW, Halliwell CE, Clark PA. 2015. Mixing length controls on high resolution simulations of convective storms. Q. J. R. Meteorol. Soc. 141: 272-284.

Istok MJ, Doviak RJ. 1986. Analysis of the relation between Doppler spectral width and thunderstorm turbulence. J. Atmos. Sci. 43: 2199-2214.

Keeler JR, Passarelli RE. 1990. Signal processing for atmospheric radars. Radar in Meteorology. 


\section{Observing turbulence in convective clouds}

DOI 10.1007/978-1-935704-15-7_21. 199-229.

Khain AP, Pinsky MB. 1995. Drops' inertia and its contribution to turbulent coalescence in convective clouds: Part 1. Drops' fall in the flow with random horizontal velocity. J. Atmos. Sci. 52: 196-206.

Knupp KR, Cotton WR. 1982. An intense, quasi-steady thunderstorm over mountainous terrain, part III: Doppler radar observations of the turbulent structure. J. Atoms. Sci. 39: 359-368.

Labitt, M. 1981. Co-ordinated radar and aircraft observations of turbulence. Project Rep. ATC 108, MIT, Lincoln Lab, 39 pp.

Lhermitte RM. 1963. Motions of scatterers and the variance of the mean intensity of weather radar signals. SRRC-RR-63-57. Sperry-Rand Res. Cent., Sudbury, Massachusetts.

Marshall JS, Palmer WM. 1948. The distribution of raindrops with size. J. Meteorol. 5: 165-166.

Meishner P, Baumann R, Holler H, Jank T. 2001. Eddy dissipation rates in thunderstorms estimated by Doppler radar in relation to aircraft in-situ measurements. J. Atmos. Oceanic. Technol. 18: $1609-1627$.

Melnikov VM, Doviak RJ. 2009. Turbulence and wind shear in layers of large Doppler spectrum width in stratiform precipitation. J. Atmos. Oceanic Technol. 26: 430-443.

Neter J, Wasserman W. 1974. Applied linear statistical models, 842pp.

Nicol JC, Hogan RJ, Stein THM, Hanley KE, Clark PA, Halliwell CE, Lean HW, Plant RS. 2015. Convective updraft evaluation in high-resolution NWP simulations using single-Doppler measurements. Q. J. R. Meteorol. Soc. 141: 3177-3189.

Pinksy MB, Khain AP. 2002. Effects of in-cloud nucleation and turbulence on droplet spectrum formation in cumulus clouds. Q. J. R. Meteorol. Soc. 128: 501-533.

Pruppacher HR, Klett JD. 1978. Microphysics of clouds and precipitation. D. Reidel Publishers, Dordrecht, 714pp.

Shupe MD, Brooks IM, Canut G. 2012. Evaluation of turbulent dissipation rate retrievals from Doppler cloud radar. Atmos. Meas. Tech. 5: 1375-1385. 


\section{Observing turbulence in convective clouds}

839

840

841

842

843

844

845

846

847

848

849

850

851

852

853

854

855

856

857

858

859

860

861

862

Stein THM, Hogan RJ, Clark PA, Halliwell CE, Hanley KE, Lean HW, Nicol JC, Plant RS. 2015. The DYMECS Project: A statistical approach for the evaluation of convective storms in highresolution NWP models. Bull. Amer. Meteor. Soc. 96: 939-951.

Ulbricht CW. 1974. Analysis of Doppler radar spectra of hail. J. Appl. Meteorol. 13: 387-396.

Vohl O, Mitra SK, Wurzler SC, Pruppacher HR. 1999. A wind tunnel study of the effects of turbulence on the growth of cloud droplets by collision and coalescence. J. Atmos. Sci. 56: 40884099.

Waldvogel A, Schmidt W, Federer B. 1978. The kinetic energy of hailfalls. Part I: Hailstone spectra. J. Appl. Meteorol. 17: 515-520.

Zrnic DS, Doviak RJ, 1989. Effect of drop oscillations on spectral moments and differential reflectivity measurements. J. Atmos. Oceanic Technol. 6: 532-536.

\section{Figure Captions}

Figure 1. (a) Change in $\sigma_{\mathrm{TV}_{j}}^{2}$ for rain, ice aggregates and hail, with radar reflectivity $Z_{j}$, and elevation angle, $\theta_{\mathrm{el}}$. Black lines refer to observations made at vertical incidence; grey lines at $\theta_{\mathrm{el}}=15^{\circ}$. (b) The impact on $\sigma_{\mathrm{TV}_{\text {hail }}}^{2}$ of using different $V_{\text {hail }}(D)$ relationships in the derivation of (13); (1) $p=$ 142.6, $q=0.5$, (2) $p=162.0, q=0.5$, (3) $p=359.0, q=0.8$. Results are displayed for $\sigma_{\mathrm{TV}}^{2}$ hail sampled at vertical incidence $\left(\theta_{\mathrm{el}}=90^{\circ}\right)$. (c) The change in $\sigma_{s \theta}^{2}, \sigma_{s \varphi}^{2}$ and $\sigma_{s r}^{2}($ Eq. (17) $-(19))$ with shear magnitude, $|S|$. Variances $\sigma_{s \theta}^{2}$, and $\sigma_{s \varphi}^{2}$ are displayed for ranges $30 \mathrm{~km}$ and $150 \mathrm{~km}$, which are roughly the minimum and maximum ranges of radar observations in the DYMECS data. Shears larger than $0.02 \mathrm{~s}^{-1}$ were uncommon in our observations. In each panel, the threshold for negligibility $\sigma_{\text {neg }}^{2}$, is plotted for reference as a dashed line at $0.5 \mathrm{~m}^{2} \mathrm{~s}^{-2}$. 


\section{Observing turbulence in convective clouds}

864

865

866

867

868

869

870

871

872

873

874

875

876

877

878

879

880

881

882

883

884

885

886

887

Figure 2. Independent distributions of $1 \times 10^{6}$ values of $S_{\varphi}$ and $S_{r}$ sourced from 31 PPI scans performed on 20 April 2012.

Figure 3. Change in the PDFs of observed $\left|S_{\varphi}\right|$ for three selected intervals of $\left|S_{r}\right|$ (solid lines).

Distributions of $\left|S_{\varphi}\right|$ are well approximated by Gamma PDFs (22) (dashed lines). The width of each $\left|S_{r}\right|$ interval is $1 \times 10^{-4} \mathrm{~s}^{-1}$, and the interval of $\left|S_{r}\right|$ for each distribution is displayed in the figure titles.

Figure 4. The change in the median, $25^{\text {th }}$ and $75^{\text {th }}$ percentile values of $\left|S_{\varphi}\right|$ with $\left|S_{r}\right|$.

Figure 5. The insensitivity of distributions of $\varepsilon$ to the scale $\Lambda_{s}$, over which shear is calculated for $\sigma_{S}^{2}$

Figure 6. Example $\varepsilon$ retrieval for an RHI scan of a convective storm performed on the 20 April 2012 (showers). Included is (a) radar reflectivity, (b) Doppler velocity, (c) vertical velocity, (d) total Doppler variance, (e) Doppler variance due to shear, and (f) eddy dissipation rate displayed in $\log _{10}$ units. The grey contour outlines reflectivity returns of $-20 \mathrm{dBZ}$.

Figure 7. Equivalent to Figure 6, an example retrieval of $\varepsilon$ for an RHI scan of a convective storm performed on the 25 August 2012 (deep convection).

Figure 8. The ratio of shear $\left(\sigma_{s}^{2}\right)$ and turbulent $\left(\sigma_{\mathrm{t}}^{2}\right)$ contributions to Doppler variance in an example shower cloud observed on 20 April 2012. The location of values of $\sigma_{s}^{2}$ that exceed $\sigma_{\text {neg }}^{2}$ is indicated by the black contour. In this example, neglecting $\sigma_{s}^{2}$ in the contoured region results in the considerable over-estimation of mean $\varepsilon$ by $52 \%$. 


\section{Observing turbulence in convective clouds}

890 Figure 9. Comparison of the vertical distribution of various percentiles of $\varepsilon$ in convective clouds 891 ( $Z>-20 \mathrm{dBZ})$ on 20 April (showers) and 25 August (deep convection), 2012. Percentiles for each $8921 \mathrm{~km}$ layer are plotted at the midpoint of that layer.

893

894

895

896

897 898

899

900

901

902

903

904 905

906

907

908 03 904

Figure 10. Scatter plots comparing the $95^{\text {th }}$ percentile of $\varepsilon$ for each updraft region $\varepsilon_{95}$, on 20 April (showers) and 25 August (deep convection), 2012, to the following corresponding statistics: (a) the $95^{\text {th }}$ percentile of vertical velocity $w_{95}$, (b) the $95^{\text {th }}$ percentile of the magnitude of the horizontal gradient in vertical velocity $\left|\frac{d w}{d x}\right|_{95}$, (c) the updraft width, and (d) the updraft depth.

Figure 11. The change in the cumulative density function (CDF) of $\varepsilon$ in updraft regions with different $95^{\text {th }}$ percentile values of $w\left(w_{95}\right)$ for 20 April 2012 shower updrafts (black lines) and 25 August 2012 deep updrafts (grey lines). Values of $w_{95}$ did not exceed $6 \mathrm{~m} \mathrm{~s}^{-1}$ in any shower updraft region. Values of $w_{95}$ smaller than $2 \mathrm{~m} \mathrm{~s}^{-1}$ were not found in any deep updraft region. 
Observing turbulence in convective clouds

909
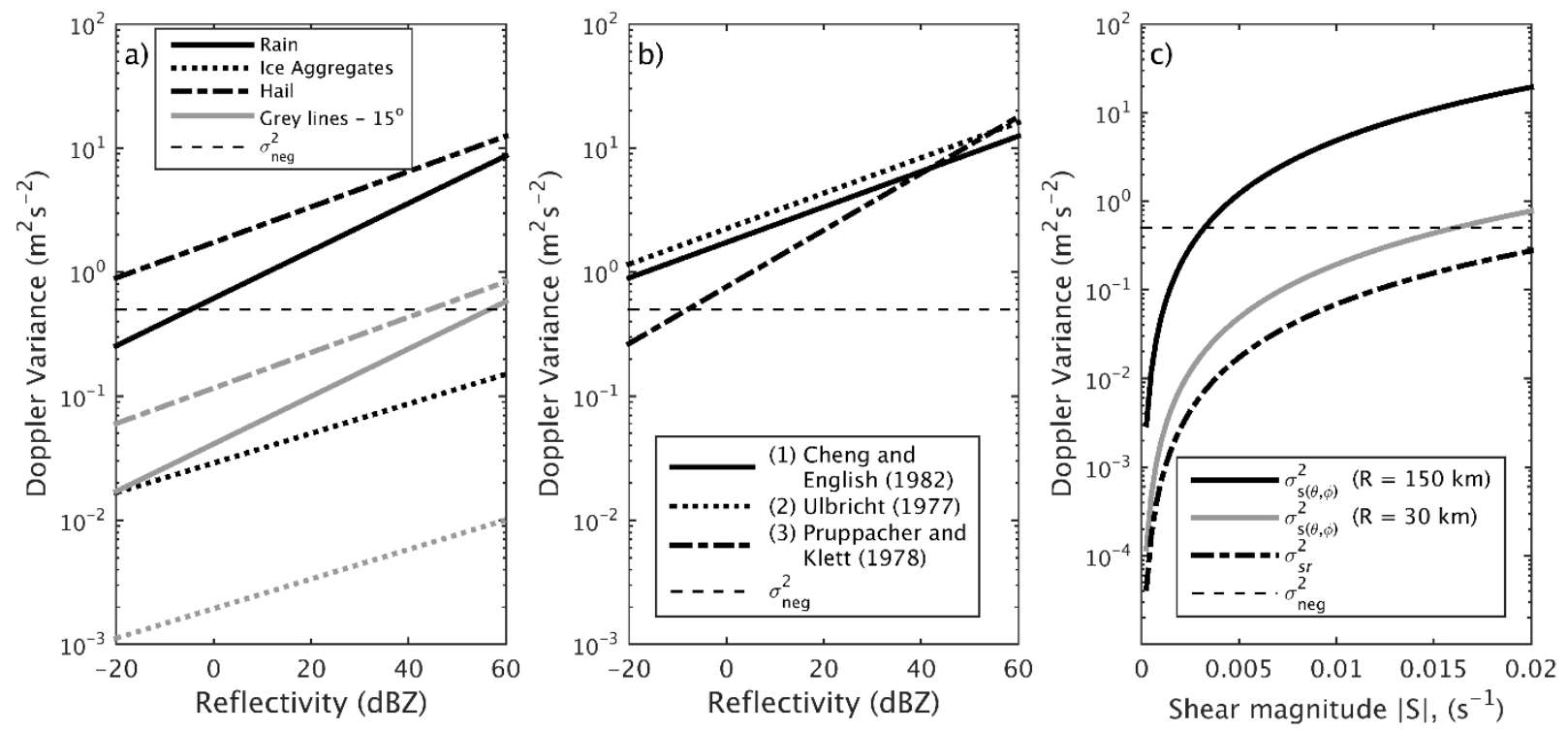

Figure 1

912

913

914

915

916

917

918

919

920

921

922

923

924 
Observing turbulence in convective clouds

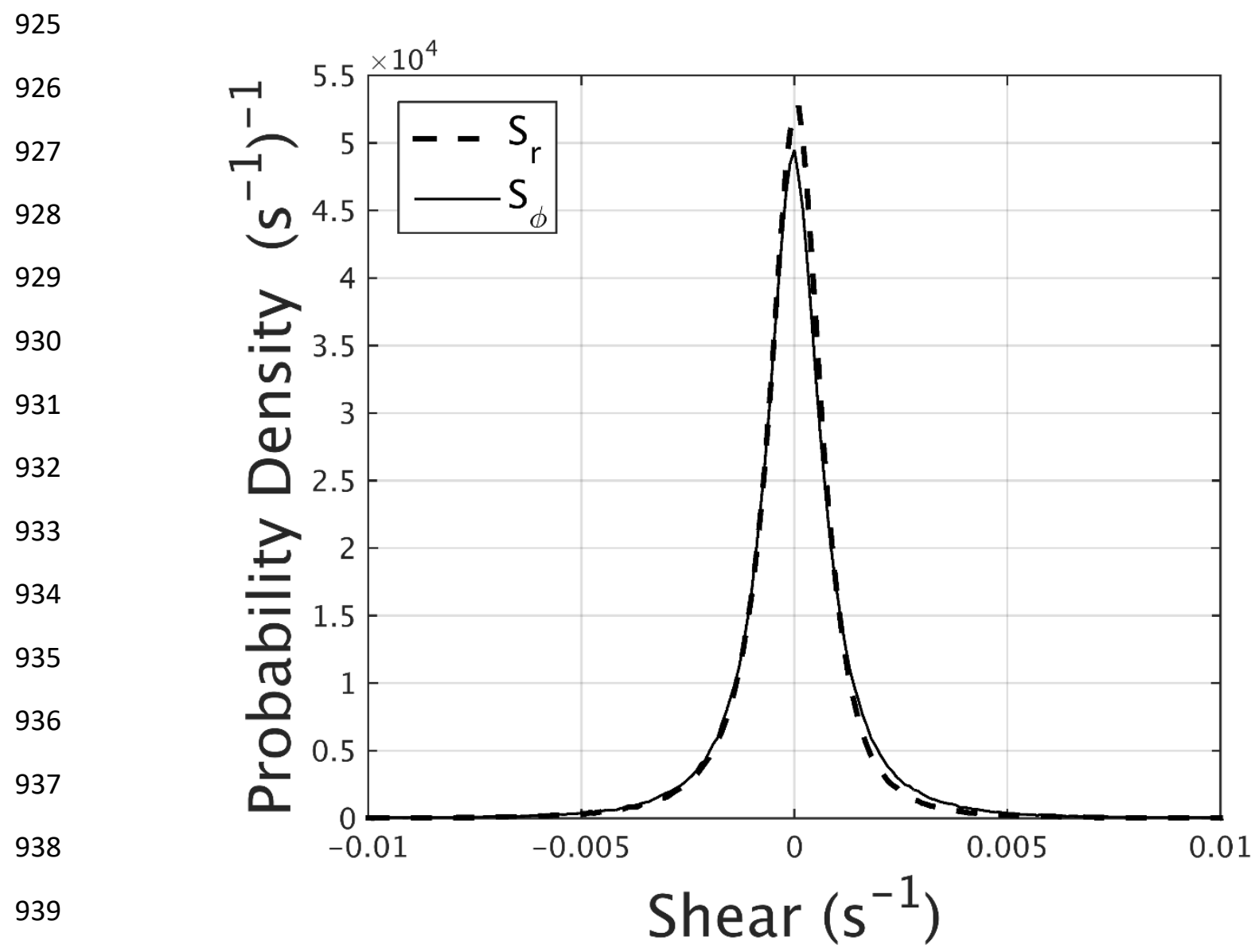

940

941

942

Figure 2

943

944

945

946

947

948

949

950

951

952

953

954

955 
Observing turbulence in convective clouds

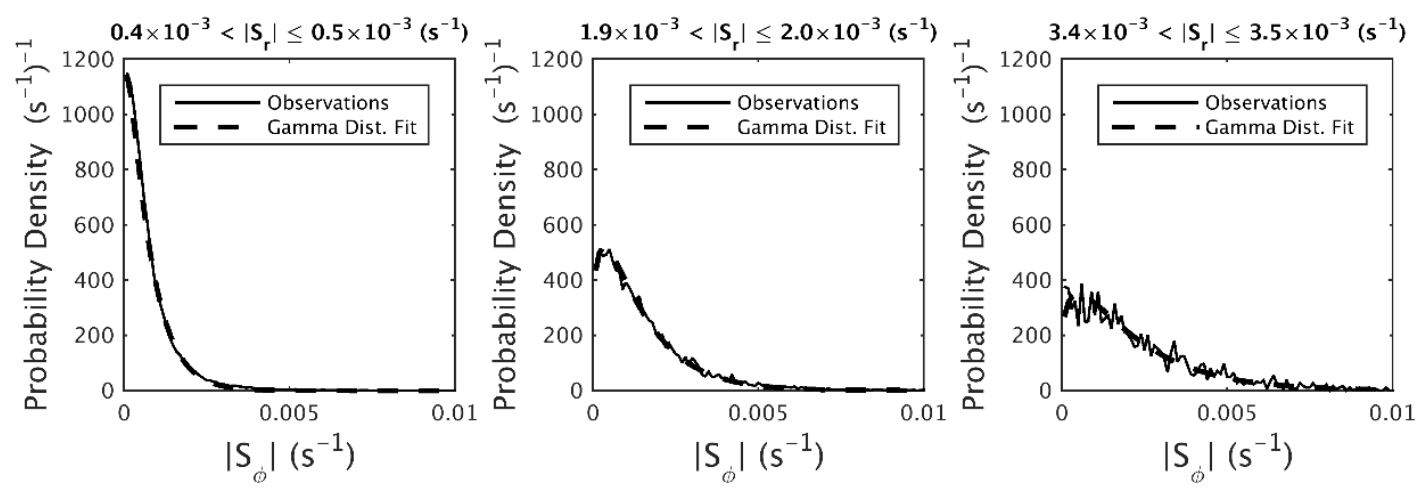

Figure 3

958

959

960

961

962

963

964

965

966

967

968

969

970

971

972

973

974

975

976

977

978 
Observing turbulence in convective clouds

979

980

981

982

983

984

985

986

987

988

989

990

991

992

993

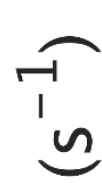

0.02

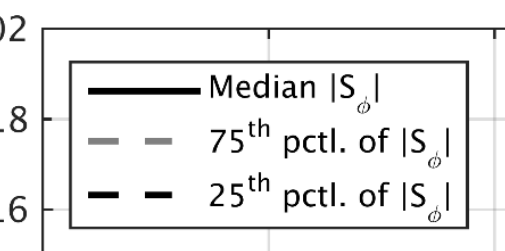

- $0.016-25^{\text {th }}$ pctl. of $\left|\mathrm{S}_{\phi}\right|$

$\frac{n}{\frac{1}{\sigma}}$

$\theta$

$\bar{\sigma}$

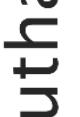

994

995

996

Figure 4

997

998

999

1000

1001

1002

1003

1004

1005

1006

1007

1008

1009 
Observing turbulence in convective clouds

1010

1011

1012

1013

1014

1015

1016

1017

1018

1019

1020

1021

1022

1023

1024

1025

1026

1027

1028

1029

1030

1031

1032

1033

1034

1035

1036

1037

1038

1039

1040

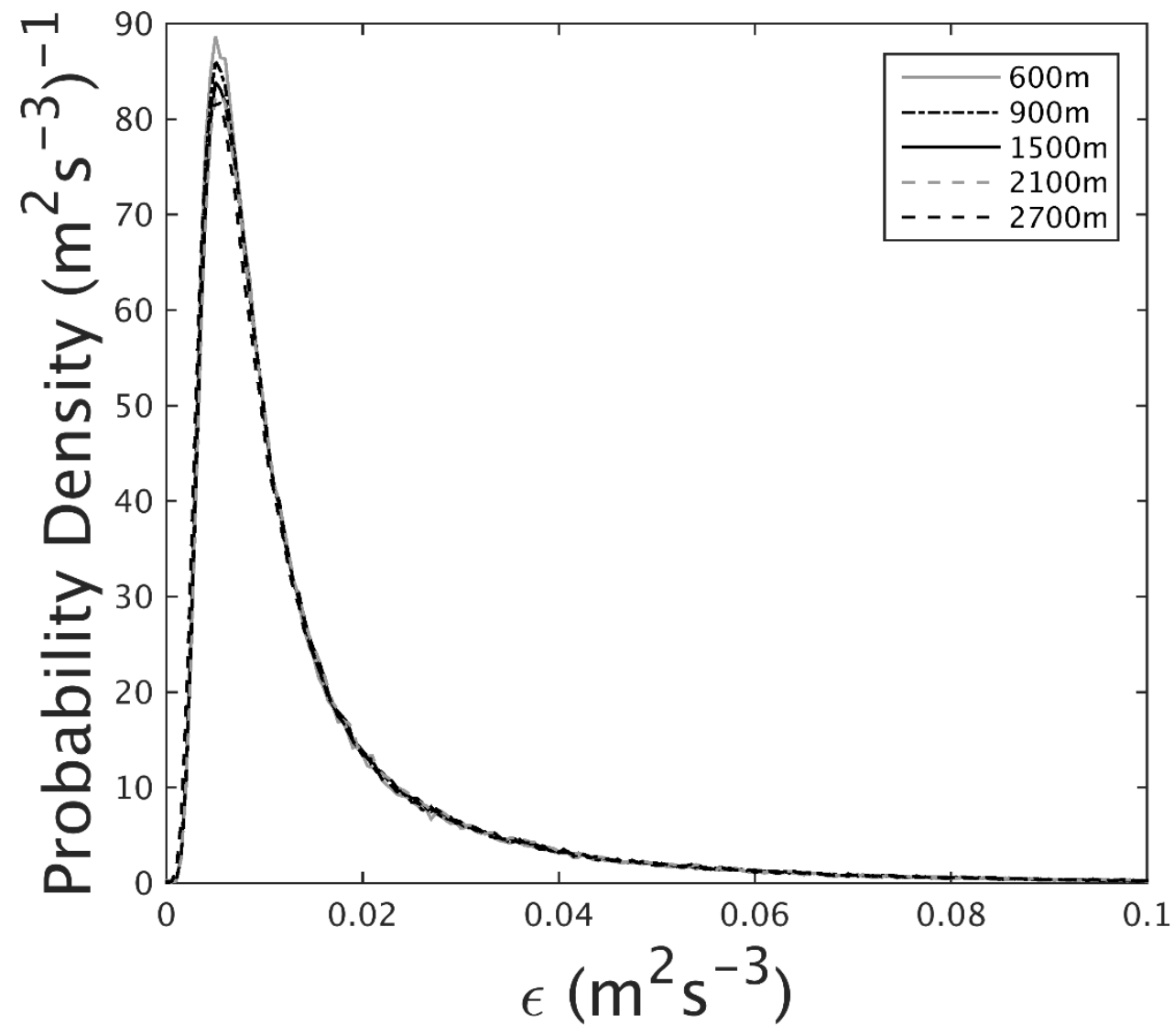

Figure 5 

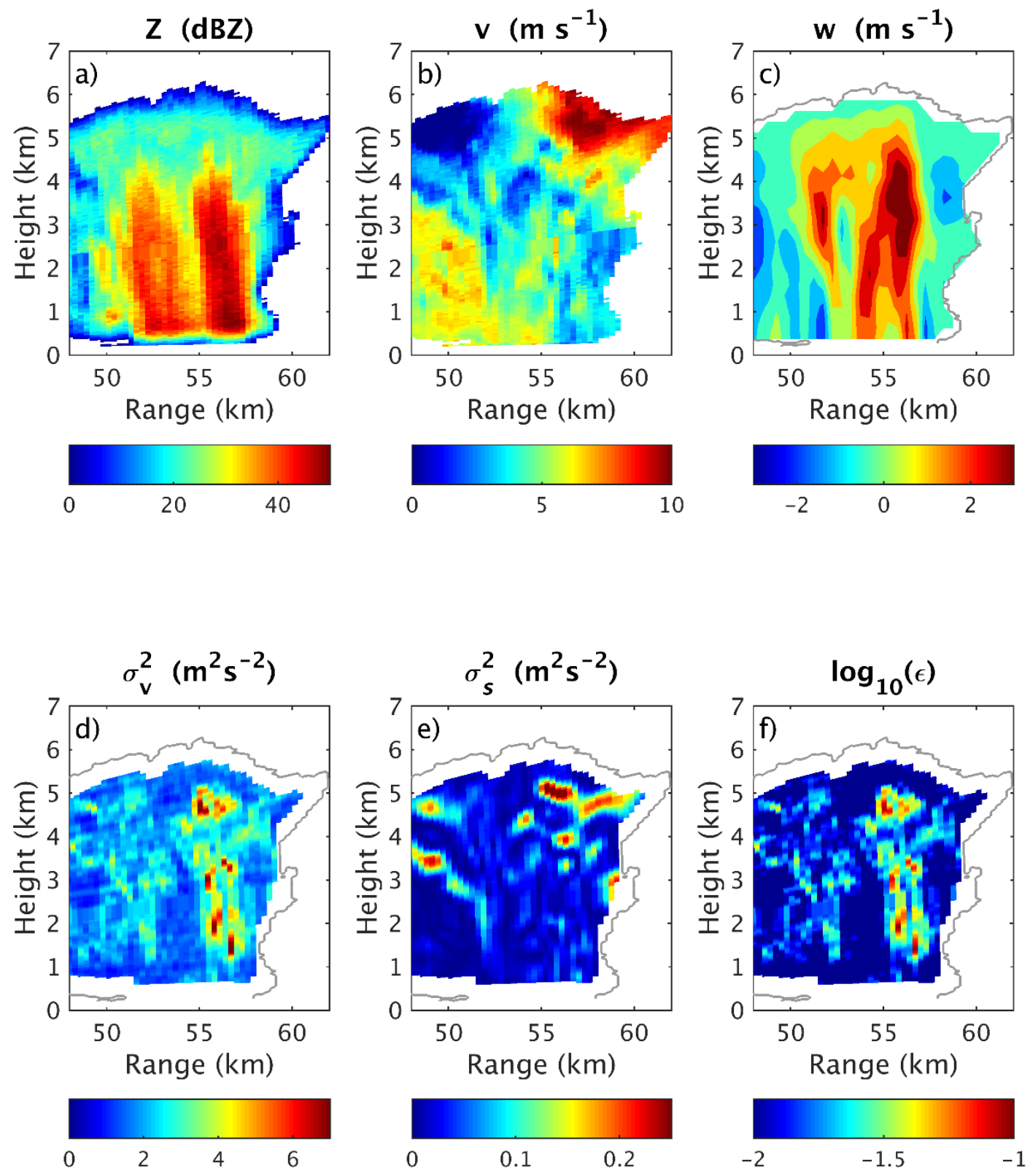

Figure 6 

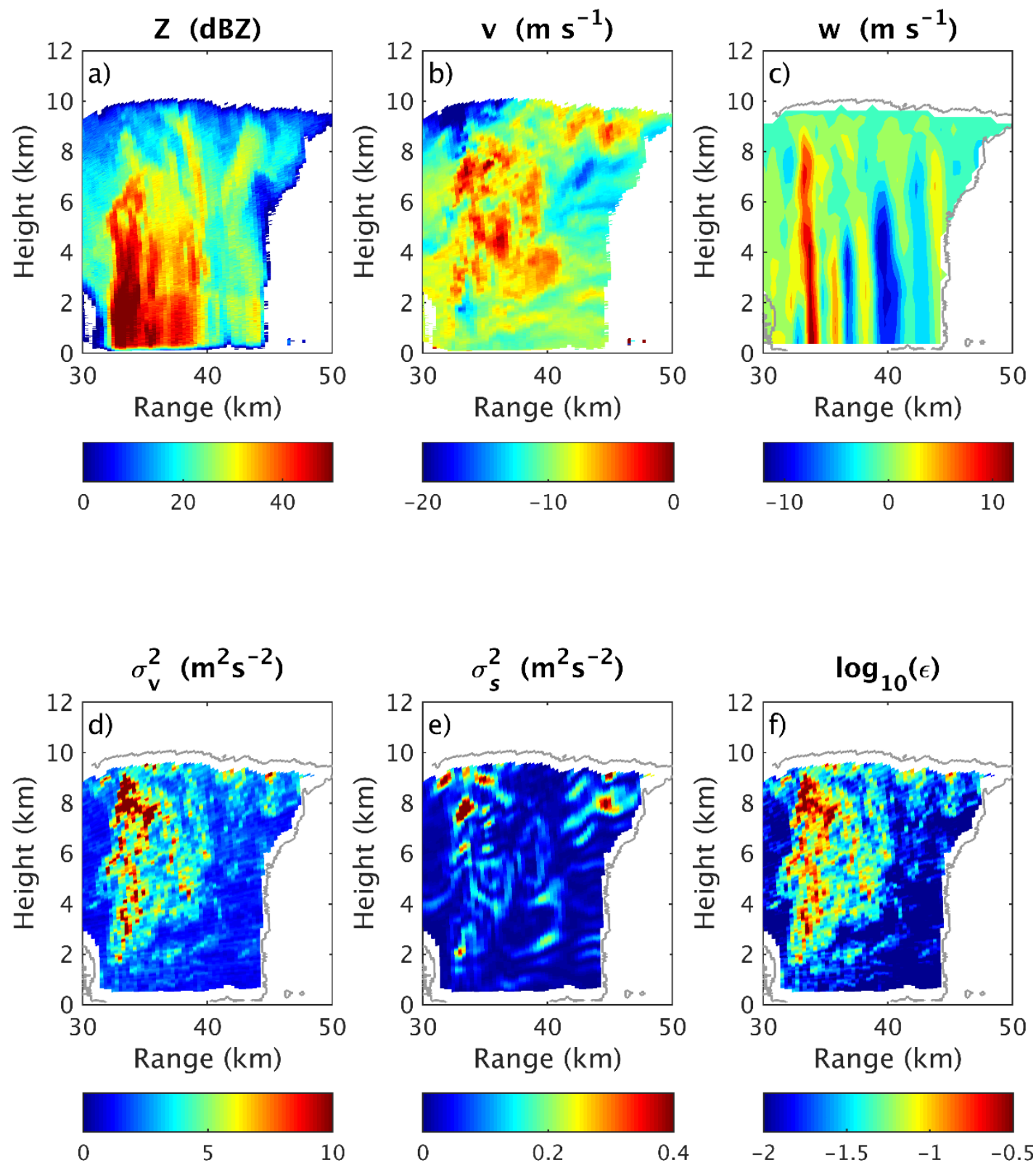

Figure 7 
Observing turbulence in convective clouds

1055

1056

1057

1058

1059

1060

1061

1062

1063

1064

1065

1066

1067

1068

1069

1070

1071

1072

1073

1074

1075

1076

1077

1078

1079

1080

1081

1082

1083

1084

1085
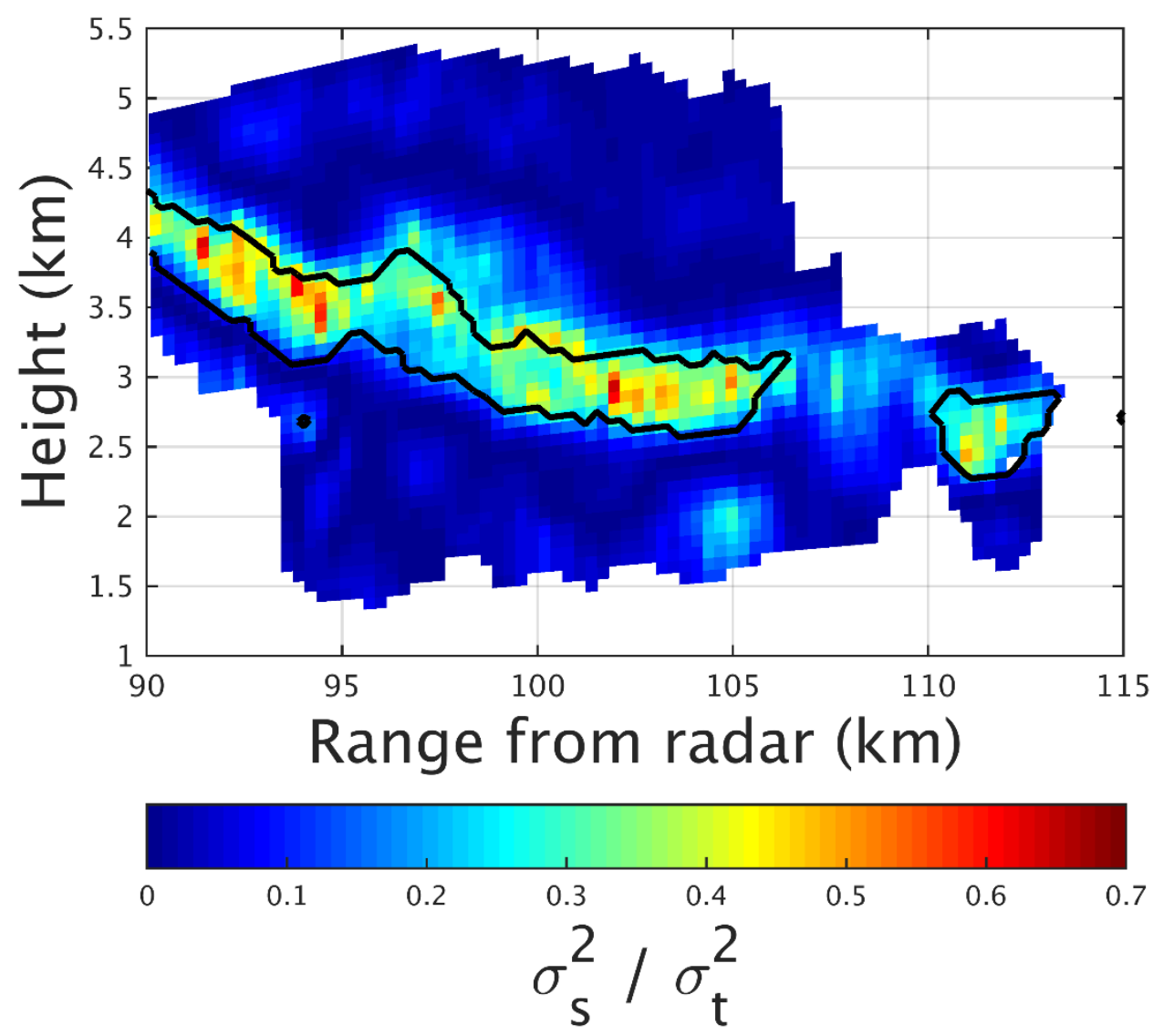

Figure 8 
Observing turbulence in convective clouds

1086

1087

1088

1089

1090

1091

1092

1093

1094

1095

1096

1097

1098

1099

1100

1101

1102

1103

1104

1105

1106

1107

1108

1109

1110

1111

1112

1113

1114

1115

1116

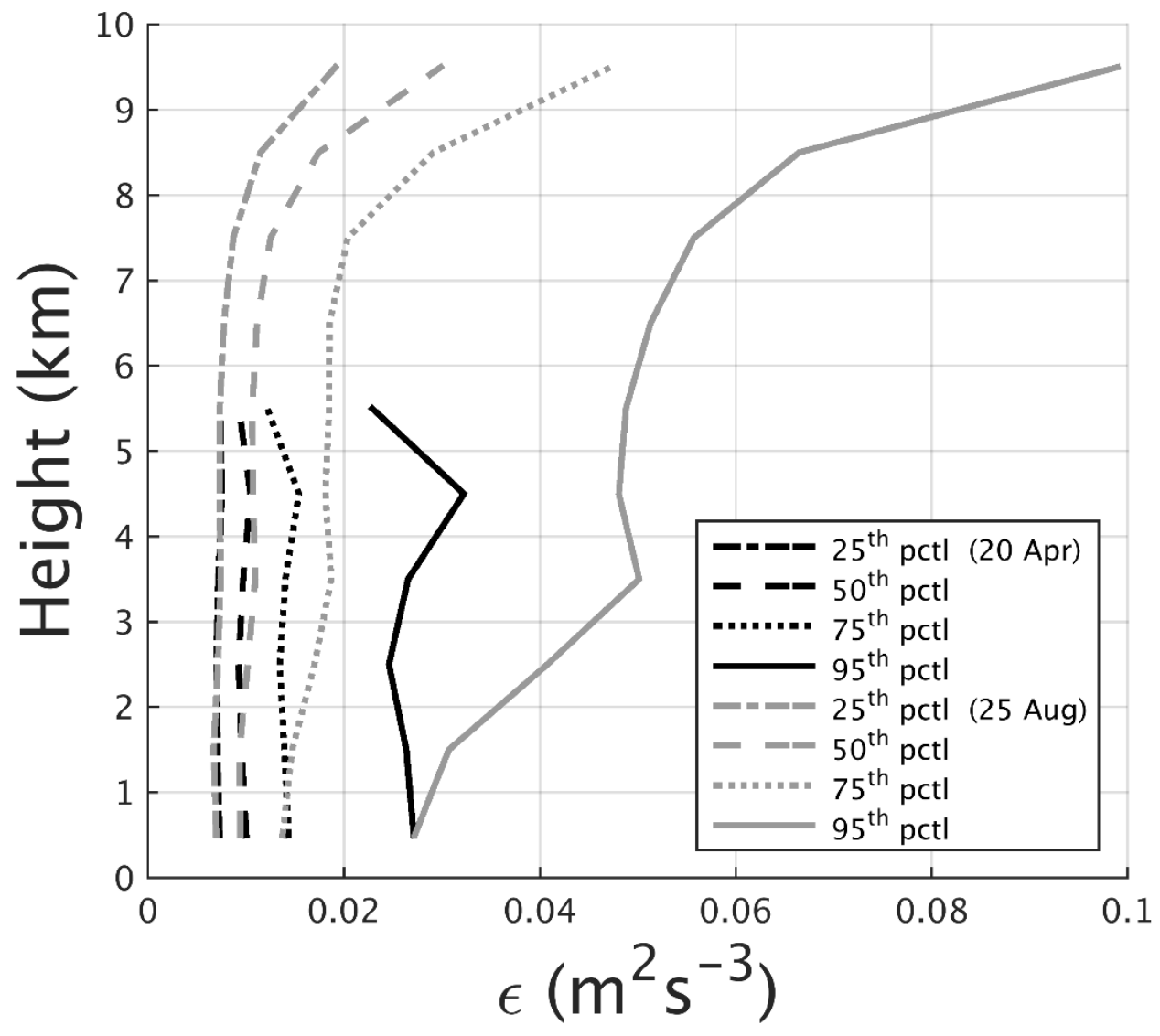

Figure 9 
Observing turbulence in convective clouds
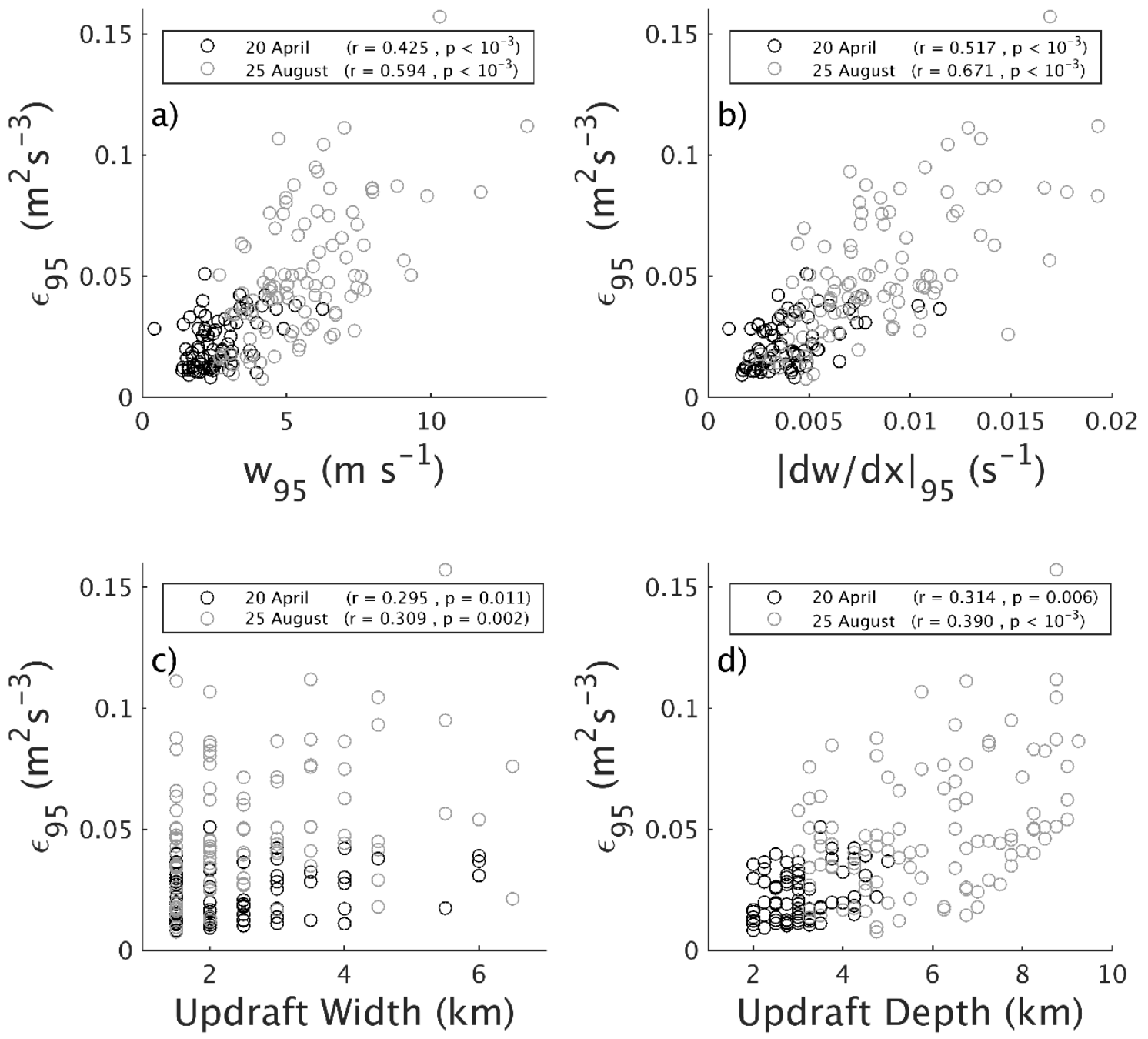

Figure 10 
Observing turbulence in convective clouds

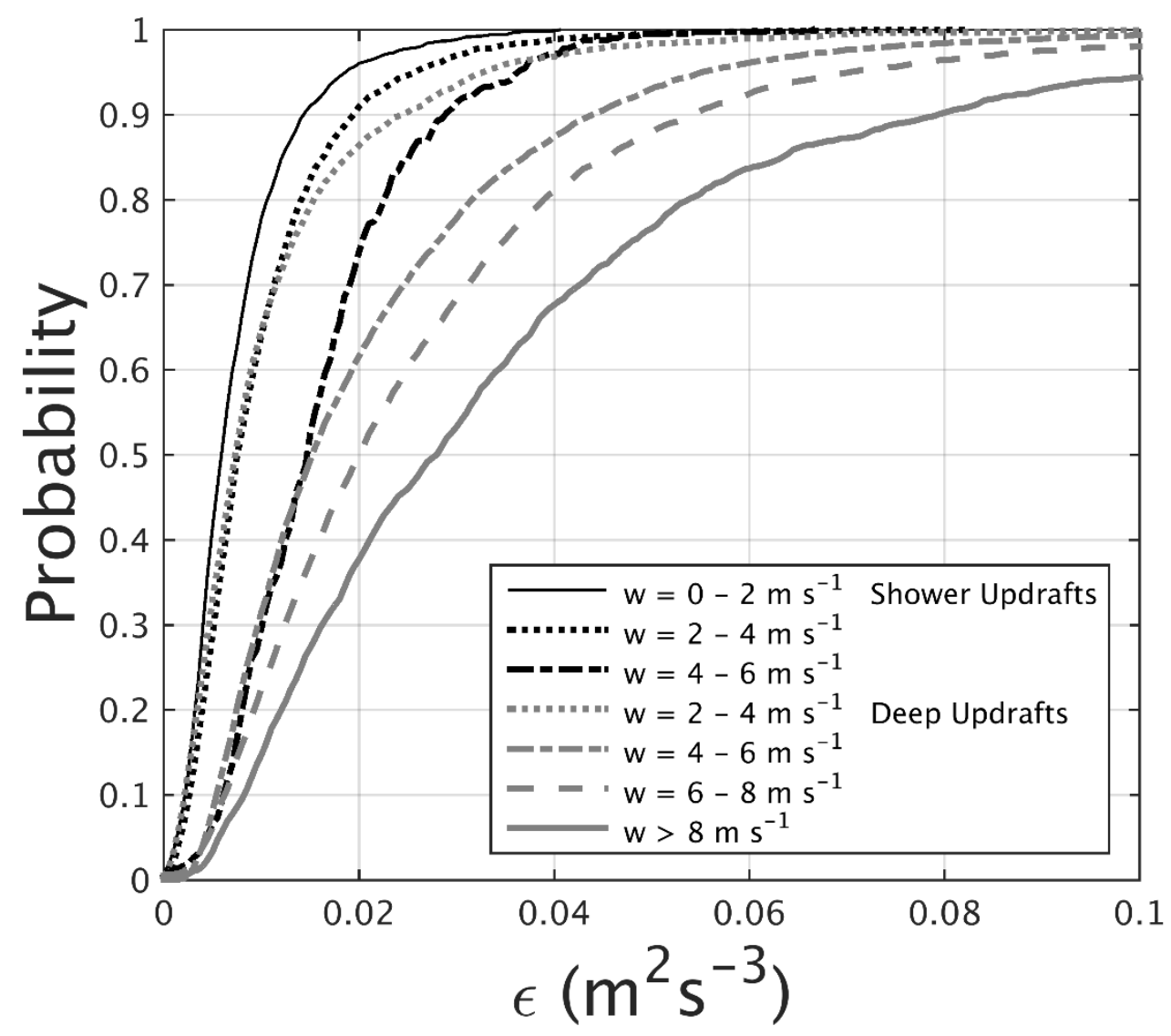

1144

Figure 11 